\title{
X-Rays from the Location of the Double-humped Transient ASASSN-15lh
}

\section{Citation}

Margutti, R., B. D. Metzger, R. Chornock, D. Milisavljevic, E. Berger, P. K. Blanchard, C. Guidorzi, et al. 2017. "X-Rays from the Location of the Double-Humped Transient ASASSN-15lh." The Astrophysical Journal 836 (1) (February 6): 25. doi:10.3847/1538-4357/836/1/25.

\section{Published Version}

doi:10.3847/1538-4357/836/1/25

\section{Permanent link}

http://nrs.harvard.edu/urn-3:HUL.InstRepos:32192694

\section{Terms of Use}

This article was downloaded from Harvard University's DASH repository, and is made available under the terms and conditions applicable to Other Posted Material, as set forth at http:// nrs.harvard.edu/urn-3:HUL.InstRepos:dash.current.terms-of-use\#LAA

\section{Share Your Story}

The Harvard community has made this article openly available.

Please share how this access benefits you. Submit a story.

\section{Accessibility}




\title{
X-Rays from the Location of the Double-humped Transient ASASSN-15lh
}

R. Margutti ${ }^{1,2}$, B. D. Metzger ${ }^{3}$, R. Chornock ${ }^{4}$, D. Milisavljevic ${ }^{5}$, E. Berger ${ }^{5}$, P. K. Blanchard ${ }^{5}$, C. Guidorzi ${ }^{6}$, G. Migliori ${ }^{7}$, A. Kamble $^{5}$, R. Lunnan ${ }^{8}$, M. Nicholl ${ }^{5}$, D. L. Coppejans ${ }^{9}$, S. Dall'Osso ${ }^{10}$, M. R. Drout ${ }^{11,14}$, R. Perna ${ }^{10}$, and B. Sbarufatti ${ }^{12,13}$

${ }^{1}$ Center for Interdisciplinary Exploration and Research in Astrophysics (CIERA) and Department of Physics and Astronomy, Northwestern University, Evanston, IL 60208, USA

${ }^{2}$ Center for Cosmology and Particle Physics, New York University, 4 Washington Place, New York, NY 10003, USA

${ }^{3}$ Columbia Astrophysics Laboratory, Columbia University, Pupin Hall, New York, NY 10027, USA

${ }^{4}$ Astrophysical Institute, Department of Physics and Astronomy, 251B Clippinger Lab, Ohio University, Athens, OH 45701, USA

${ }^{5}$ Harvard-Smithsonian Center for Astrophysics, 60 Garden Street, Cambridge, MA 02138, USA

${ }^{6}$ Department of Physics and Earth Sciences, University of Ferrara, via Saragat 1, I-44122, Ferrara, Italy

${ }^{7}$ Laboratoire AIM (CEA/IRFU-CNRS/INSU-Université Paris Diderot), CEA DSM/SAp, F-91191 Gif-sur-Yvette, France

${ }^{8}$ Department of Astronomy, California Institute of Technology, 1200 East California Boulevard, Pasadena, CA 91125, USA

${ }^{9}$ Department of Astrophysics/IMAPP, Radboud University, P.O. Box 9010, 6500 GL Nijmegen, The Netherlands

${ }^{10}$ Department of Physics and Astronomy, Stony Brook University, Stony Brook, NY, 11794, USA

${ }^{11}$ Carnegie Observatories, 813 Santa Barbara Street, Pasadena, CA 91101, USA

12 Department of Astronomy and Astrophysics, Pennsylvania State University, 525 Davey Lab, University Park, PA 16802, USA

${ }^{13}$ INAF, Osservatorio Astronomico di Brera, via E. Bianchi 46, I-23807 Merate, Italy

Received 2016 September 27; revised 2016 November 27; accepted 2016 November 28; published 2017 February 6

\begin{abstract}
We present the detection of persistent soft X-ray radiation with $L_{x} \sim 10^{41}-10^{42} \mathrm{erg} \mathrm{s}^{-1}$ at the location of the extremely luminous, double-humped transient ASASSN-15lh as revealed by Chandra and Swift. We interpret this finding in the context of observations from our multiwavelength campaign, which revealed the presence of weak narrow nebular emission features from the host-galaxy nucleus and clear differences with respect to superluminous supernova optical spectra. Significant UV flux variability on short timescales detected at the time of the rebrightening disfavors the shock interaction scenario as the source of energy powering the long-lived UV emission, while deep radio limits exclude the presence of relativistic jets propagating into a low-density environment. We propose a model where the extreme luminosity and double-peaked temporal structure of ASASSN-15lh is powered by a central source of ionizing radiation that produces a sudden change in the ejecta opacity at later times. As a result, UV radiation can more easily escape, producing the second bump in the light curve. We discuss different interpretations for the intrinsic nature of the ionizing source. We conclude that, if the $\mathrm{X}$-ray source is physically associated with the optical-UV transient, then ASASSN-15lh most likely represents the tidal disruption of a main-sequence star by the most massive spinning black hole detected to date. In this case, ASASSN-15lh and similar events discovered in the future would constitute the most direct probes of very massive, dormant, spinning, supermassive black holes in galaxies. Future monitoring of the X-rays may allow us to distinguish between the supernova hypothesis and the hypothesis of a tidal disruption event.
\end{abstract}

Key words: supernovae: individual (ASASSN-15lh)

Supporting material: machine-readable table

\section{Introduction}

Optical surveys sampling the sky over timescales of a few days significantly advanced our knowledge of astronomical transients of different origins, including superluminous supernovae (SLSNe; Chomiuk et al. 2011; Quimby et al. 2011; GalYam 2012), very fast-rising stellar explosions (e.g., Drout et al. 2014), and stellar tidal disruption events (TDEs) caused by supermassive black holes (Rees 1988; Komossa 2015). Occasionally, a transient is found with properties that seem to defy all previous classification schemes. The event ASASSN$15 \mathrm{lh}$ belongs to this category.

ASASSN-15lh (Dong et al. 2016) was discovered by the AllSky Automated Survey for Supernovae (ASAS-SN ${ }^{15}$ ) on 2015 June 14 at $z=0.2326(d=1171 \mathrm{Mpc}$ for standard Planck cosmology). Its extremely large peak luminosity $L_{\mathrm{pk}} \sim 2 \times 10^{45} \mathrm{erg} \mathrm{s}^{-1}$ and the blue, almost featureless spectrum with no apparent sign of $\mathrm{H}$ or $\mathrm{He}$ (and some

\footnotetext{
${ }^{14}$ Hubble, Carnegie-Dunlap Fellow.

15 http://www.astronomy.ohio-state.edu/assassin/index.shtml
}

spectroscopic resemblance to the SLSN 2010gx) led Dong et al. (2016) and Godoy-Rivera et al. (2016) to suggest that ASASSN-15lh is the most luminous SLSN ever detected. The very large energy radiated by ASSASN $-15 \mathrm{lh}\left(E_{\mathrm{rad}} \sim(1.5-2 \times\right.$ $10^{52} \mathrm{erg}$, Godoy-Rivera et al. 2016) requires extreme properties of the progenitor star and sources of energy that are different from the standard radioactive decay of ${ }^{56} \mathrm{Ni}$ that powers normal $\mathrm{H}$-stripped SNe in the local universe (Chatzopoulos et al. 2016; Dong et al. 2016; Kozyreva et al. 2016; van Putten \& Della Valle 2017). In this context, the double-humped light curve of ASASSN-15lh has been interpreted by Chatzopoulos et al. (2016) as a signature of the interaction of massive SN ejecta $M_{\mathrm{ej}} \sim 36 M_{\odot}$ with an H-poor circumstellar shell of $M_{\text {CSM }} \sim 20 M_{\odot}$, possibly supplemented by radiation from a newly born rapidly rotating magnetar (Metzger et al. 2015; Bersten et al. 2016; Dai et al. 2016; Sukhbold \& Woosley 2016).

The old, massive $M_{*} \sim 2 \times 10^{11} M_{\odot}$ host galaxy of ASASSN-15lh, with limited star formation rate (SFR $<0.3 M_{\odot} \mathrm{yr}^{-1}$; Dong et al. 2016), however, is markedly different from the host galaxies of core-collapse $\mathrm{SNe}$ (e.g., 
Leaman et al. 2011) as well as of envelope-stripped SLSNe, which tend to be younger star-forming systems with significantly lower stellar mass (Lunnan et al. 2014, 2015; Leloudas et al. 2016; Perley et al. 2016). This observation, together with the location of the transient-astrometrically consistent with the host-galaxy nucleus-inspired a connection between ASASSN-15lh and the tidal disruption of a star by the supermassive black hole (SMBH) in the host galaxy (Brown et al. 2016b; Godoy-Rivera et al. 2016; Leloudas et al. 2016; Perley et al. 2016). In this context ASASSN-15lh would be the most luminous TDE ever observed, associated with an SMBH with mass $M_{\bullet} \sim 10^{8.6} M_{\odot}$ (Dong et al. 2016; Godoy-Rivera et al. 2016), significantly larger than any SMBH currently associated with a TDE (e.g., Komossa 2015).

It is clear that the luminosity, the spectral properties, and the double-humped light curve of ASASSN-15lh, as well as its host galaxy, are unprecedented both in the context of SLSNe and in the context of TDEs.

In this paper we present and discuss the following observational facts: (i) the uncovering of persistent, soft $\mathrm{X}$-ray emission at the location of ASASSN-15lh (Sections 2.1 and 2.2); (ii) the detection of significant temporal variability at UV wavelengths during the rebrightening phase (Section 2.3); (iii) the detection of narrow nebular spectral features connected to the host-galaxy nucleus (Section 2.4). We propose a scenario where a single physical mechanism can naturally explain the double-humped light curve of ASASSN-15lh and suggest that its location-very close to or coincident with the nucleus of a galaxy that harbors an SMBH-is likely the key to unlocking the mysterious nature of the transient (Section 3). Conclusions are drawn in Section 4.

In our analysis we assume the object's time of first light to be 2015 April 29, corresponding to a 30 day (rest-frame) rise time to maximum $V$-band luminosity (Dong et al. 2016). Our main conclusions do not depend on this assumption.

\section{Data Analysis and Results}

\subsection{X-Ray Analysis: $C X O$}

We obtained four epochs of deep X-ray observations of ASASSN-15lh with the Chandra X-ray Observatory $(C X O)$ on 2015 November 12 (exposure of $10 \mathrm{ks}$ ), 2015 December 13 (10 ks), 2016 February 20 (40 ks), and 2016 August 19 (30 ks, PI Margutti), corresponding to $\delta t=129.4$ days, $\delta t=154.6$, $\delta t=210.5$ days, and $\delta t=357.8$ days rest-frame since optical maximum light, which occurred on 2015 June 5 (Dong et al. 2016). CXO data have been reduced with the CIAO software package (version 4.8) and corresponding calibration files. Standard ACIS data filtering has been applied.

ASASSN-15lh is not detected in our first epoch of observations (ID 17879), with a $3 \sigma$ upper limit on the count rate of $9.98 \times 10^{-5}$ counts s$^{-1}(0.5-8 \mathrm{keV})$. The Galactic column density in the direction of the transient is $3.07 \times 10^{20} \mathrm{~cm}^{-2}$ (Kalberla et al. 2005). For an assumed power-law spectrum with photon index $\Gamma=2$ and Galactic absorption, the unabsorbed $0.3-10 \mathrm{keV}$ flux limit is $F_{x}<1.1 \times 10^{-15} \mathrm{erg} \mathrm{s}^{-1} \mathrm{~cm}^{-2} \quad\left(L_{x}<1.8 \times 10^{41} \mathrm{erg} \mathrm{s}^{-1}\right)$. Our analysis below favors a soft X-ray spectrum with negligible absorption and $\Gamma \sim 3$ or a thermal spectrum with $T \sim 0.17 \mathrm{keV}$. For these parameters, the unabsorbed $0.3-10 \mathrm{keV}$ flux limit is $F_{x}<2.0 \times 10^{-15} \mathrm{erg} \mathrm{s}^{-1} \mathrm{~cm}^{-2}$
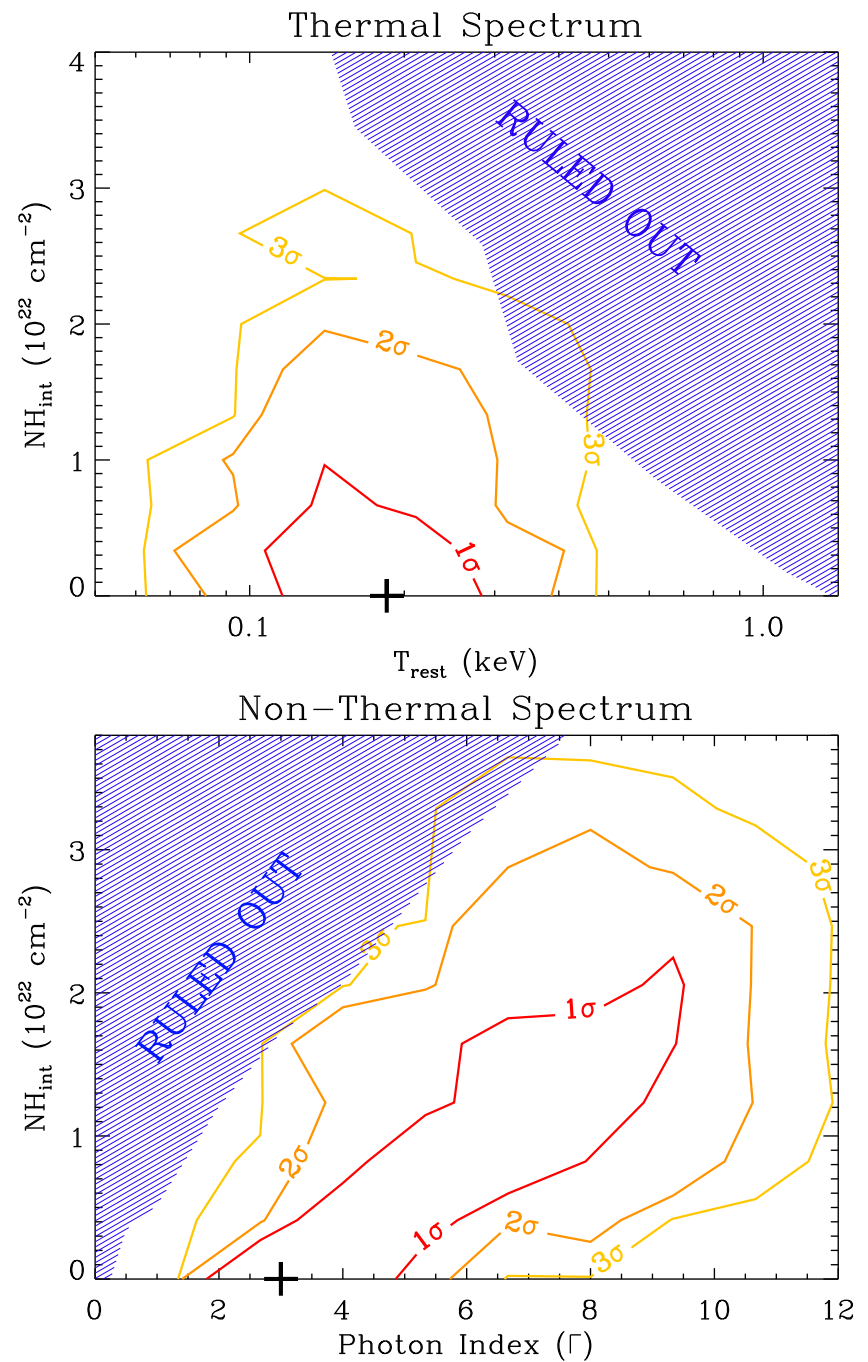

Figure 1. Constraints on the spectral model parameters of the X-ray source detected at the location of ASASSN-15lh, as derived from our second epoch (shaded region) and a joint fit of the third and fourth epochs (contours) of $C X O$ observation. Upper panel: absorbed blackbody spectrum. Lower panel: absorbed power-law spectrum. In both panels the cross symbol identifies the best-fitting model parameters. These observations favor a soft X-ray spectrum with limited intrinsic absorption.

(nonthermal spectrum) and $F_{x}<8.0 \times 10^{-16} \mathrm{erg} \mathrm{s}^{-1} \mathrm{~cm}^{-2}$ (thermal spectrum).

In our second epoch of observation (ID 17880) we find evidence for weak, soft X-ray emission at the location of ASASSN-15lh. We detect two photons with energy $<1 \mathrm{keV}$ in a $1^{\prime \prime}$ region around the transient, corresponding to a detection at $4.3 \sigma$ confidence level in the energy range $0.5-1 \mathrm{keV}$, and to a detection at $3.3 \sigma$ confidence level in the range $0.5-8 \mathrm{keV}$. We constrain the spectral parameters by using the observed background and the actual instrumental response to simulate the expected emission from a grid of thermal and nonthermal spectral models with a wide range of intrinsic absorption $\mathrm{NH}_{\mathrm{int}}=(0-4) \times 10^{22} \mathrm{~cm}^{-2}$. The regions excluded at $3 \sigma$ confidence are shaded in Figure 1.

An X-ray source is clearly detected at the location of ASASSN-15lh at the time of our third $C X O$ observation (ID 17881 ), with count rate $1.5 \times 10^{-4}$ counts $\mathrm{s}^{-1}$ and significance of $5.2 \sigma$ in the range $0.5-8 \mathrm{keV}(4.7 \sigma$ in the energy range $0.5-1 \mathrm{keV}$ ). In our fourth epoch (ID 17882) the source is still 
detected with count rate $1.7 \times 10^{-4}$ counts $\mathrm{s}^{-1}$ and significance of $4.9 \sigma$ in the range $0.5-8 \mathrm{keV}(3.6 \sigma$ in the energy range $0.5-1 \mathrm{keV}$ ).

We employ the Cash statistics to fit the spectra (we have a total of six and five photons in a $1^{\prime \prime}$ region around the transient in the third and fourth epochs, respectively), and perform a series of Markov chain Monte Carlo simulations to constrain the spectral parameters. The analysis of each of the two epochs taken separately points to a soft X-ray spectrum, with limited absorption and no evidence for statistically significant evolution between the two epochs. We thus constrain the spectral parameters of the X-ray source with a joint spectral fit of the two $C X O$ epochs of observation, where the spectral normalization is allowed to vary from one epoch to the other.

The results are displayed in Figure 1. For an absorbed, nonthermal power-law spectrum, the best-fitting parameters are $\Gamma=3.0$ and $\mathrm{NH}_{\text {int }} \sim 10^{19} \mathrm{~cm}^{-2}$. The inferred $(0.3-10 \mathrm{keV})$ unabsorbed flux for this model is $F_{x}=3.6 \times 10^{-15} \mathrm{erg} \mathrm{s}^{-1} \mathrm{~cm}^{-2}$, corresponding to $L_{x}=5.9 \times 10^{41} \mathrm{erg} \mathrm{s}^{-1}$ (third epoch) and $F_{x}=$ $4.9 \times 10^{-15} \mathrm{erg} \mathrm{s}^{-1} \mathrm{~cm}^{-2}\left(L_{x}=8.1 \times 10^{41} \mathrm{erg} \mathrm{s}^{-1}\right.$, fourth epoch). The best-fitting parameters for an absorbed blackbody spectrum are $T=0.17 \mathrm{keV}$ and $\mathrm{NH}_{\mathrm{int}} \sim 10^{18} \mathrm{~cm}^{-2}$. The inferred $(0.3-10 \mathrm{keV})$ unabsorbed flux for this model is $F_{x}=1.2 \times$ $10^{-15} \mathrm{erg} \mathrm{s}^{-1} \mathrm{~cm}^{-2}$, corresponding to $L_{x}=2.0 \times 10^{41} \mathrm{erg} \mathrm{s}^{-1}$ (third epoch) and $F_{x}=1.4 \times 10^{-15} \mathrm{erg} \mathrm{s}^{-1} \mathrm{~cm}^{-2}\left(L_{x}=2.3 \times\right.$ $10^{41} \mathrm{erg} \mathrm{s}^{-1}$, fourth epoch). Both spectral models point to a very limited amount of neutral hydrogen in the host galaxy along our line of sight, consistent with the very low $N(\mathrm{HI})$ inferred by Leloudas et al. (2016) from Ly $\alpha$ and the very strong highionization lines ( $\mathrm{N} \mathrm{V}$ and $\mathrm{O} \mathrm{VI}$ ).

With reference to Figure 1 we find that: (i) the X-ray source shows a soft spectrum (most of the allowed parameter space is at $\Gamma>2$ and $T<1 \mathrm{keV}$ ) with limited intrinsic absorption (of the order of a few $10^{22} \mathrm{~cm}^{-2}$ at most); (ii) there is no evidence for strong temporal and/or spectral variability of the X-ray source.

We first evaluate the possibility that the X-ray emission arises from a population of low-mass X-ray binaries (LMXBs) residing in the early-type host galaxy, using the relations $L_{x}-$ $L_{B}$ and $L_{x}-L_{K}$ of Kim \& Fabbiano (2004). For the host galaxy of ASASSN-15lh Dong et al. (2016) measure $M_{K}=-25.5$ mag and $M_{B}=-19.96$ mag, which imply $L_{x}$, $\mathrm{XRB}=(1-6) \times 10^{40} \mathrm{erg} \mathrm{s}^{-1}(0.3-8 \mathrm{keV})$. This is a factor $\geqslant 10$ smaller than the measured $\mathrm{X}$-ray emission at the location of ASASSN-15lh (recalibrated with the same spectral model as Kim \& Fabbiano (2004) in the $0.3-8 \mathrm{keV}$ band). We conclude that LMXBs are unlikely to be the source of the detected $\mathrm{X}$-rays. We thus envision two possible scenarios: either the $\mathrm{X}$-rays originate from weak activity from the host-galaxy nucleus or they are physically connected to the optical/UV transient. In the first case we expect a somewhat stable X-ray emission over the timescale of years, while we anticipate fading if the X-ray emission is directly connected to ASASSN-15lh. Future observations will clarify the origin of the detected highenergy emission. Below we put our results into the context of $\mathrm{X}$-ray emission from known transients (i.e., SNe and TDEs).

The detected emission is softer than the typical X-ray spectrum of $\mathrm{SNe}$ associated with gamma-ray bursts (GRBs) ( $\Gamma \sim 2$, e.g., Margutti et al. 2013b) and normal H-stripped SNe (e.g., Chevalier \& Fransson 2006; Dwarkadas \& Gruszko 2012), which typically show $\Gamma \sim 2$ and a decaying flux with time. A way to sustain luminous X-ray emission over a long time is to invoke the $\mathrm{SN}$ shock interaction with a thick medium (see, e.g., SN 2014C). However, the observed X-ray spectrum of H-stripped SNe strongly interacting with the environment is even harder $(T \sim 20 \mathrm{keV})$, and thus even more different from what we observe at the location of ASASSN-15lh. It is thus unlikely that a $\mathrm{SN}$ shock interaction with the medium is powering both the X-ray and optical/UV emission from ASASSN-15lh. Finally, compared to the only other X-ray source associated with a Type I SLSN (SLSN-I) so far, the emission at the location of ASASSN-15lh is also softer and significantly longer lived (Figure 2): for the SLSN-I SCP06F6, Levan et al. (2013) reports $\Gamma \sim 2.6$ (or a thermal spectrum with $T \sim 1.6 \mathrm{keV})$.

The X-ray properties of ASASSN-15lh are instead more reminiscent of the soft X-ray emission detected in non-jetted TDEs. Non-jetted TDEs detected with ROSAT, XMM-Newton, Chandra, and more recently with Swift show peak luminosities of $L_{x} \sim 10^{42}-10^{44} \mathrm{erg} \mathrm{s} \mathrm{s}^{-1}$ and very soft spectra that later harden with time on a timescale of years and with initial temperatures $T<0.2 \mathrm{keV}$ (e.g., Komossa 2015 for a recent review).

Just like the TDEs ASASSN-15oi (Holoien et al. 2016b) and ASASSN-14li (van Velzen et al. 2016), the X-ray emission is more luminous than what is expected based on the extrapolation of the optical/UV blackbody model (see Section 2.3) and a more complex model is needed. In this context ASASSN-15lh would show the most extreme ratio $L_{\nu, \mathrm{UV}} / L_{\nu, \mathrm{X} \text {-rays }} \sim 10^{5}$ (compared to $L_{\nu, \mathrm{UV}} / L_{\nu, \mathrm{X} \text {-rays }} \sim 10^{2-3}$ for ASASSN-14li and $L_{\nu, \mathrm{UV}} / L_{\nu, \mathrm{X} \text {-rays }} \sim 10^{4}$ for ASASSN-15oi).

In Figure 2 we put ASASSN-15lh on the X-ray luminosity plane of energetic envelope-stripped core-collapse $\mathrm{SNe}$ (i.e., GRB-SNe and SLSNe) and TDEs. ASASSN-15lh is $\sim 1000$ times less luminous than the SLSN-I SCP06F6 and does not experience a similar drop in luminosity. At $\sim 100$ days, the $\mathrm{X}$-ray emission at the location of ASASSN-15lh is more luminous than GRB-SNe. However, observations obtained around the same epoch by the ATCA in Figure 3 put deep limits on the radio emission from ASASSN-15lh (Kool et al. 2015; Leloudas et al. 2016), and rule out the presence of powerful jets seen on-axis (most of the parameter space associated with off-axis GRB-like jets is also ruled out). Also in this case, the luminous and not strongly variable X-ray emission at the location of ASASSN-15lh, which lacks a luminous radio counterpart, seems to be more in line with observations of non-jetted TDEs (recent examples are ASASSN-14li, Miller et al. 2015; Alexander et al. 2016; Holoien et al. 2016b; or ASASSN-15oi, Holoien et al. 2016a).

\subsection{X-Ray Analysis: Swift/XRT and XMM-Newton}

We reprocessed all the X-ray data collected by the Swift-XRT (Burrows et al. 2005) between 2015 June 24 and 2016 July 22 (total exposure time of $\sim 270 \mathrm{ks}$ ), following the prescriptions outlined in Margutti et al. (2013b). A targeted search for X-ray emission at the location of ASASSN-15lh identifies the presence of a weak X-ray excess with significance of $3 \sigma$ in the range $0.3-5 \mathrm{keV}$. The significance is reduced to $2.4 \sigma$ in the energy range $0.3-10 \mathrm{keV}$, consistent with the soft X-ray spectrum suggested by the $C X O$ observations. We infer a backgroundsubtracted count rate of $(1.1 \pm 0.4) \times 10^{-4}$ counts s$^{-1}$ $(0.3-5 \mathrm{keV})$, which corresponds to an unabsorbed $0.3-10 \mathrm{keV}$ flux $F_{x}=(4.1 \pm 1.5) \times 10^{-15} \mathrm{erg} \mathrm{s}^{-1} \mathrm{~cm}^{-2}$ and $F_{x}=(3.7 \pm 1.4) \times 10^{-15} \mathrm{erg} \mathrm{s}^{-1} \mathrm{~cm}^{-2}$ for blackbody and 


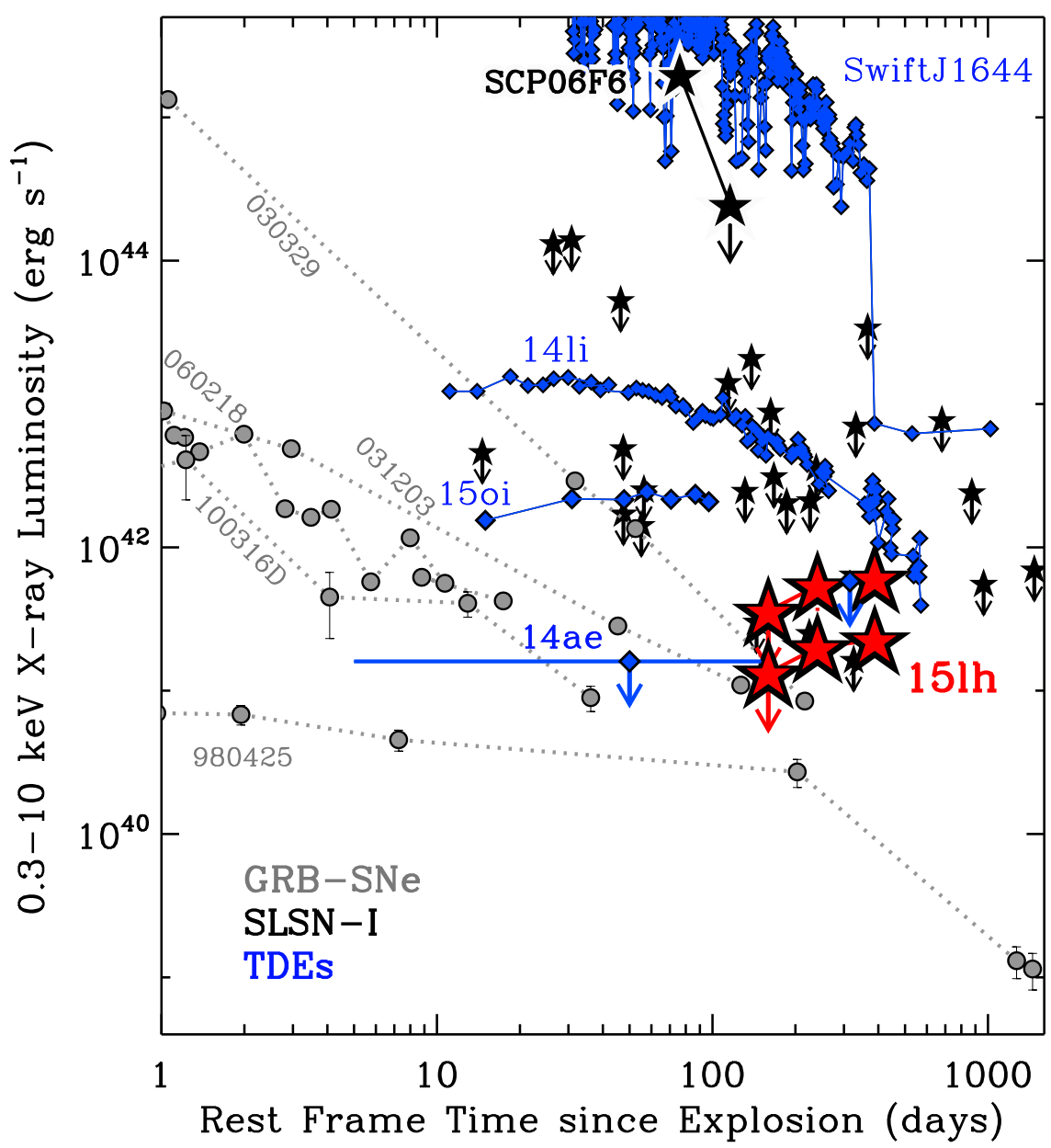

Figure 2. ASASSN-15lh in the X-ray phase space of envelope-stripped SNe and TDEs. For ASASSN-15lh we merged the second and third $C X O$ epochs for the sake of clarity and we show the luminosity of ASASSN-15lh at each epoch for both a thermal (bottom points) and a nonthermal (top points) spectrum. At $\sim 100$ days, ASASSN-15lh is more luminous than any previously detected H-stripped SN with the exception of SCP06F6. Its persistent and soft X-ray emission is more similar to nonrelativistic TDEs, like ASASSN-15oi and ASASSN-14li. The X-ray light curve of the relativistic TDE SwiftJ2058 overlaps with that of the TDE SwiftJ1644 (Pasham et al. 2015) and it is not displayed here for clarity. References: Gezari et al. (2012), Margutti et al. (2013a), Holoien et al. (2014), Miller et al. (2015), Holoien et al. (2016b), Mangano et al. (2016), Brown et al. (2016a). For SLSNe-I we updated the sample of Levan et al. (2013). The detailed analysis will appear in R. Margutti et al. (2017, in preparation).

power-law spectral models, respectively, and the best-fitting spectral parameters derived from the $C X O$ data. The average flux inferred from Swift-XRT observations is thus consistent with the results from the $C X O$ analysis and suggests that the $\mathrm{X}$-ray source at the location of ASASSN-15lh experienced at most mild temporal variability over the $\sim 1 \mathrm{yr}$ of Swift monitoring. We note that flux variations of the order of a factor of a few are consistent with our findings, given the uncertainties affecting both the Swift-XRT and the $C X O$ measurements. The X-ray source is not significantly detected if we divide the Swift-XRT data into "before" and "after" the UV rebrightening, suggesting that some level of emission is contained in both intervals. A delayed onset of the X-ray emission with respect to the optical emission is also clearly allowed, since Swift-XRT data started to be collected after optical maximum light.

XMM-Newton observed ASASSN-15lh on 2015 November $18(\delta t=134.2$ days rest-frame since maximum light), six days after our first $C X O$ epoch, which yielded a nondetection. We analyzed the $X M M$ data using standard routines in the Scientific Analysis System (SAS version 15.0.0) and the relative calibration files. We employ a source region of $32^{\prime \prime}$ radius and extract the background from a source-free region on the same chip. No X-ray source is detected at the location of ASASSN15lh. Our best constraints are derived from observations obtained with EPIC-MOS2, with total exposure time of $9 \mathrm{ks}$ (after removal of time windows contaminated by proton flaring) and a $3 \sigma$ upper limit on the count rate of 0.002 counts s$^{-1}$ $(0.3-10 \mathrm{keV})$. For the best-fitting spectral models derived from $C X O$ detections, we infer the following unabsorbed $0.3-10 \mathrm{keV}$ flux limits: $F_{x}<1.4 \times 10^{-14} \mathrm{erg} \mathrm{s}^{-1} \mathrm{~cm}^{-2}$ and $F_{x}<1.5 \times 10^{-14} \mathrm{erg} \mathrm{s}^{-1} \mathrm{~cm}^{-2}$ for the blackbody and powerlaw spectra, respectively. $X M M$ observations do not reach the necessary depth to probe the emission from the X-ray source that we detect with $C X O$ and the stacking of Swift-XRT observations. A summary of the results from the X-ray observations of ASASSN-15lh can be found in Table 1.

Finally, we comment on the $X M M$ results from Leloudas et al. (2016). From the same $X M M$ observations, Leloudas et al. (2016) infer a flux limit at 95\% confidence level of $F_{x}<2 \times 10^{-16} \mathrm{erg} \mathrm{s}^{-1} \mathrm{~cm}^{-2}$ in the range $0.3-1 \mathrm{keV}$. We do not confirm these results. From our analysis above and the spectral parameters inferred from our $C X O$ detections, we infer a $3 \sigma$ flux limit $F_{x}<1.3 \times 10^{-14} \mathrm{erg} \mathrm{s}^{-1} \mathrm{~cm}^{-2}$ and $F_{x}<1.1 \times 10^{-14} \mathrm{erg} \mathrm{s}^{-1} \mathrm{~cm}^{-2}$ for the blackbody and power- 


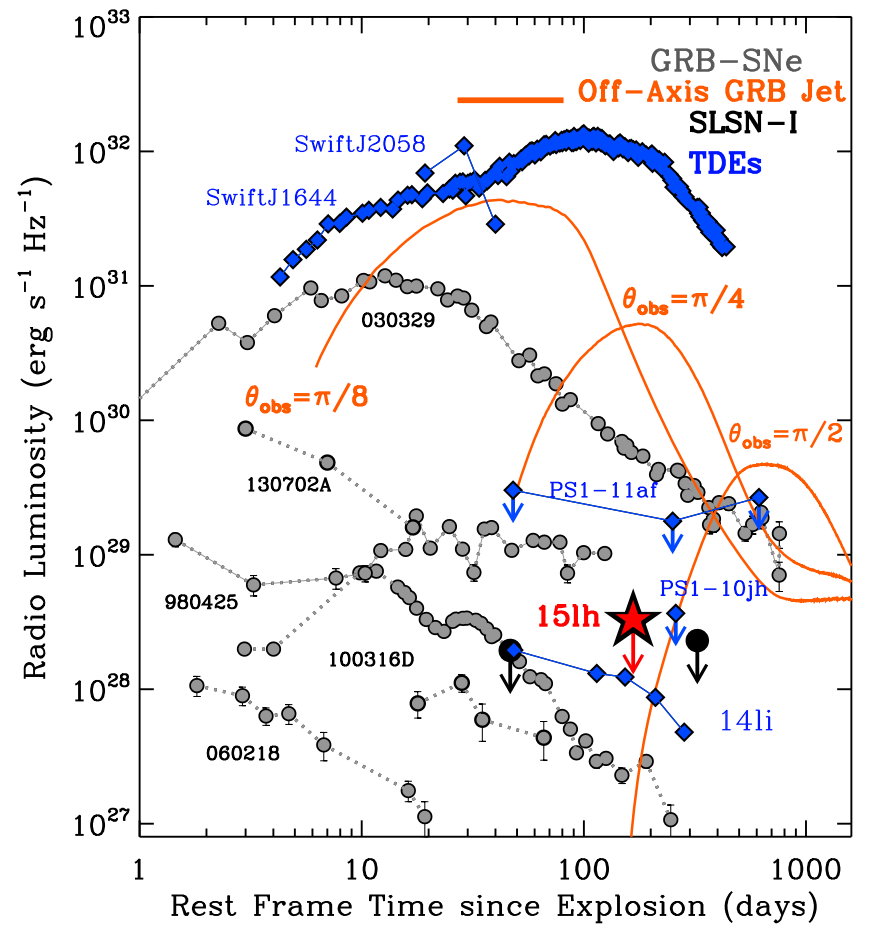

Figure 3. ASASSN-15lh (red star) in the context of radio emission from envelope-stripped SNe (gray and black filled circles for GRB-SNe and SLSNe, respectively) and TDEs (blue diamonds), as constrained by observations with ATCA (Kool et al. 2015). Radio observations acquired by Leloudas et al. (2016) three weeks later led to very similar limits and are not displayed here for clarity. The limits on the radio emission from ASASSN-15lh rule out most of the parameter space associated with powerful relativistic jets with kinetic energy $E_{k}=10^{51} \mathrm{erg}$ propagating into a circumburst medium with density $1 \mathrm{~cm}^{-3}$ (orange lines, van Eerten et al. 2010). SwiftJ1644 and SwiftJ2058 are the two relativistic TDEs known to date with radio observations. References: Cenko et al. (2012), Chomiuk et al. (2012), Chandra \& Frail (2012), van Velzen et al. (2013), Margutti et al. (2013a), Chornock et al. (2014), Nicholl et al. (2016), Alexander et al. (2016), Berger et al. (2012), Pasham et al. (2015), Zauderer et al. (2013).

law spectra, respectively, in the $0.3-1 \mathrm{keV}$ energy band. The very deep flux limit reported by Leloudas et al. (2016) originates from the extremely soft spectral model (i.e., blackbody with $T=18 \mathrm{eV}$ ) used for the X-ray flux calibration. This model is confidently ruled out by our $C X O$ detections (Figure 1), and the flux inferred from this model is thus not realistic.

\subsection{UV Analysis}

We reanalyzed all the Swift-UVOT observations obtained from 2015 June 24 until 2016 July 22 following the prescriptions by Brown et al. (2009) and adopting the updated calibration files and revised zero points of Breeveld et al. (2011). Each individual frame has been visually inspected and quality-flagged. Observations with insufficient exposure time have been merged to obtain higher signal-to-noise ratio $(\mathrm{S} / \mathrm{N})$ images from which we extracted the final photometry (Table 2). We corrected for Galactic extinction in the direction of the transient $(E(B-V)=0.03 \mathrm{mag}$, Schlafly \& Finkbeiner 2011) and subtracted the host-galaxy flux component as constrained by Dong et al. (2016). We performed a self-consistent flux calibration, and applied a dynamical conversion from count to flux that accounts for the spectral evolution of ASASSN-15lh, following the procedure outlined in Margutti et al. (2014a). Finally, we computed a

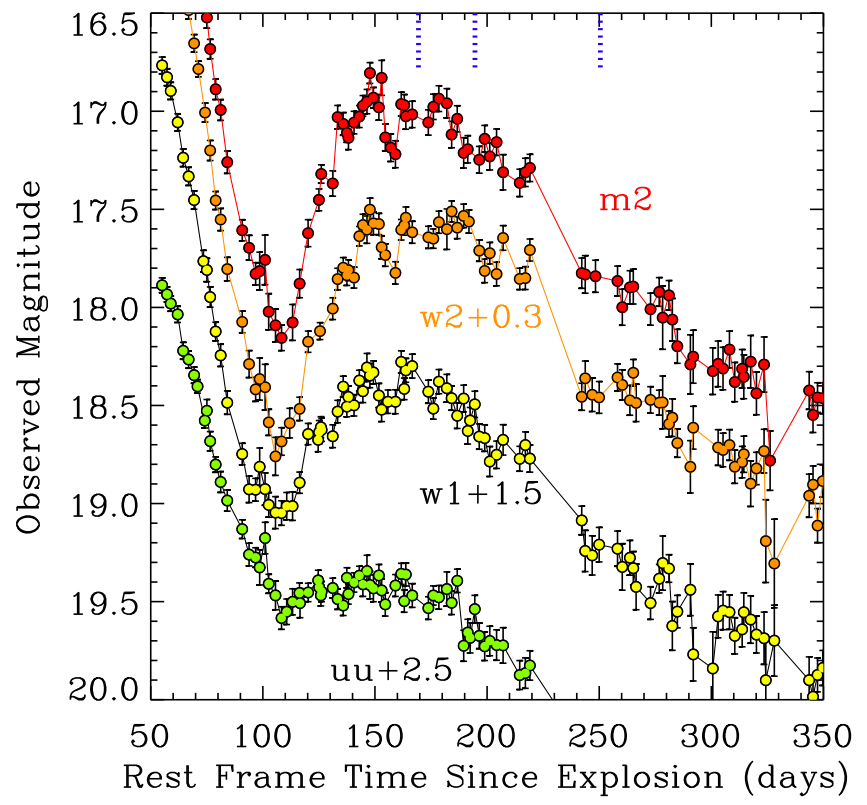

Figure 4. Swift-UVOT observations of ASASSN-15lh reveal the presence of pronounced time variability on short timescales $\Delta t \lesssim 5$ days across the SwiftUVOT bands at the time of the rebrightening. Vertical dotted lines mark the times of the $C X O$ observations.

bolometric light curve of ASASSN-15lh by integrating the best-fitting blackbody spectra.

A partial collection of the Swift-UVOT photometry of ASASSN-15lh has already been presented by Dong et al. (2016), Godoy-Rivera et al. (2016), Brown et al. (2016b), and Leloudas et al. (2016). Here we update the observations and focus on the presence of significant temporal variability that appears at the time of the rebrightening. Figure 4 shows the presence of pronounced temporal variability across the UVOT bands, and it is more pronounced at UV wavelengths as first noticed by Brown et al. (2016b). The short variability timescale $\Delta t \lesssim 5$ days at $\sim 150$ days since first light argues against the interpretation of the SN shock interaction with the surroundings as the main source of energy powering the rebrightening (Chatzopoulos et al. 2016), unless the circumstellar medium (CSM) is clumpy and with structure on short length scales. For a typical SN shock velocity $v_{\mathrm{sh}} \sim 0.2 c$ (e.g., Margutti et al. 2014b, their Figure 2) we do not expect significant temporal variability on $\Delta t<30$ days at $t \sim 150$ days, contrary to what we observe in ASASSN-15lh. This observation motivates us to consider alternative explanations for the UV rebrightening (Section 3.1).

\subsection{Late-time Optical Spectroscopy}

We acquired deep multi-epoch optical spectroscopy of ASASSN-15lh, spanning the time range $\delta t=35-350$ restframe days after maximum light and sampling key points in the late evolution of the transient. A more detailed analysis will be presented in future work (R. Chornock et al. 2016, in preparation). Here we concentrate on an analysis of our highest $\mathrm{S} / \mathrm{N}$ late-time spectrum, which was acquired well after the second rebrightening and when the underlying emission from the stellar population of the host galaxy is better revealed.

We observed ASASSN-15lh on 2016 June $10(\delta t=301$ days rest-frame since maximum light) using the Low 


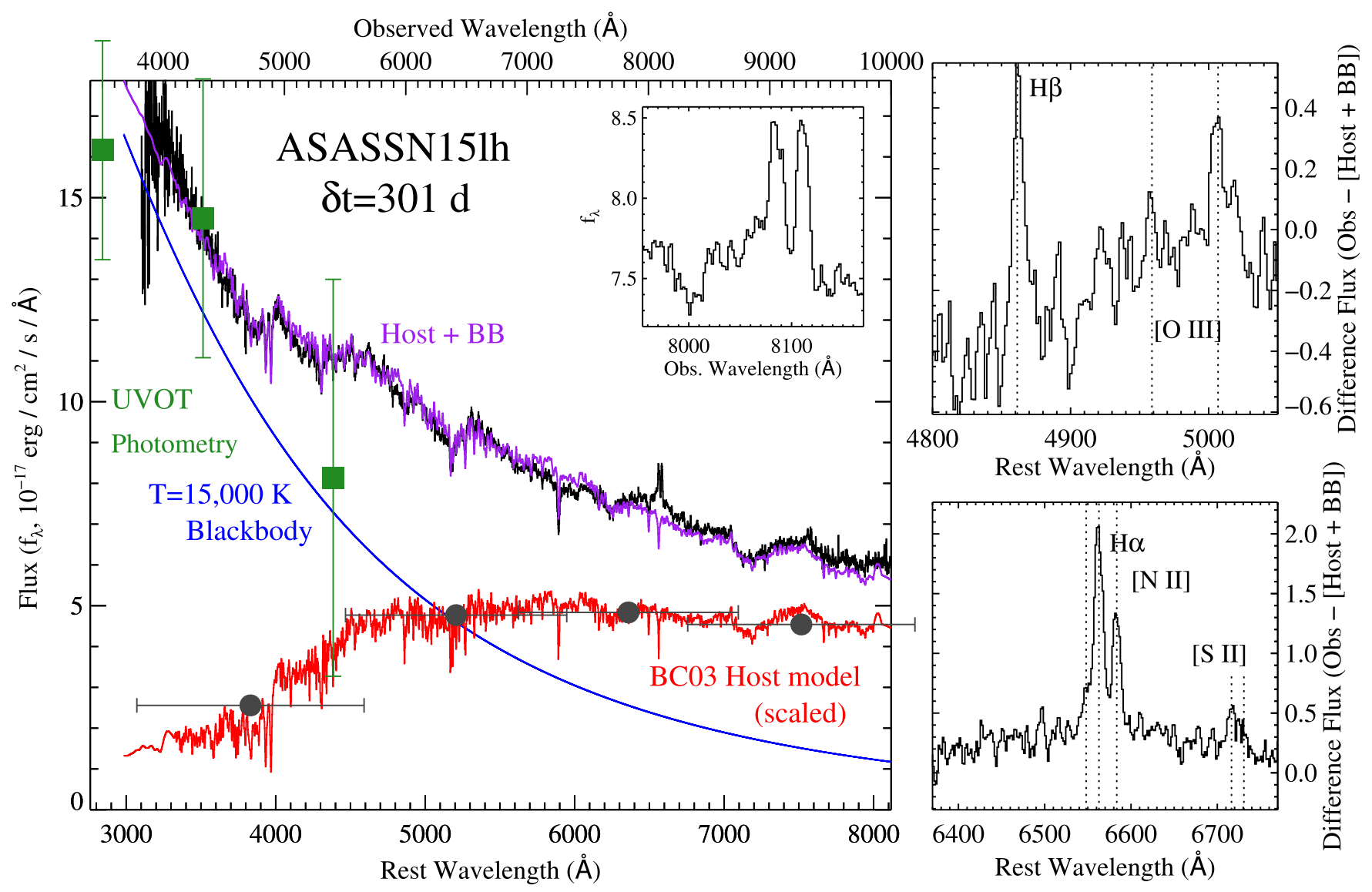

Figure 5. Left panel: a decomposition of the observed late-time spectrum (black line) obtained on 2016 June 10 ( $\delta t=301$ days rest-frame since maximum light) into a sum (magenta line) of the FAST host-galaxy model that best fits the pre-transient photometry (red line) and a featureless blackbody with temperature inferred from our fits of ASASSN-15lh (blue line). This demonstrates that the strongest spectral features can be reasonably attributed to the underlying stellar population of the host galaxy. The green squares mark the Swift-UVOT photometry after subtraction of the host model and interpolated to the date of observation. The gray circles are the scaled values for the broadband photometry of the host (Dong et al. 2016). We emphasize the lack of unambiguous evidence for broad spectral features associated with the transient. Inset: the observed spectrum in the vicinity of the $\mathrm{H} \alpha /[\mathrm{N} \mathrm{II}]$ complex is shown enlarged. Two clear emission lines are present prior to any correction for the stellar continuum. Right panels: zoom-ins to spectral regions of interest in the difference spectrum show the presence of narrow emission lines at the expected wavelengths (dotted lines) associated with $\mathrm{H} \alpha, \mathrm{H} \beta$, [N II] $\lambda \lambda 6548,6583$, and possibly [S II] and [O III] $\lambda 5007$ at a common redshift of $z=0.2320$.

Dispersion Survey Spectrograph (LDSS3C; Allington-Smith et al. 1994) on the $6.5 \mathrm{~m}$ Magellan Clay telescope. We obtained three 1800 s exposures using the VPH-All grism and a $1^{\prime \prime}$ wide slit near the center of the field of view oriented at a position angle of $128^{\circ} .3$, which was close to the parallactic angle (Filippenko 1982). This setup covered the range $3800-10500 \AA$ with a resolution of $8.1 \AA$. Standard IRAF $^{16}$ tasks were used to perform two-dimensional image processing. We used custom IDL scripts to perform flux calibration and correction for telluric absorption using observations of EG131 obtained immediately prior to those of the object. We took particular care to mitigate the effects of second-order light contamination by combining observations of the standard star taken both with and without an order-blocking filter. However, small residual contamination at long wavelengths $(\lambda>8000 \AA)$ is possible.

The resulting spectrum is shown in black in Figure 5. Numerous stellar absorption features from starlight in the host galaxy are visible, as well as two emission peaks near $\mathrm{H} \alpha$ (observed wavelengths $\sim 8100 \AA$ ). Several authors have fit the

\footnotetext{
${ }^{16}$ IRAF is distributed by the National Optical Astronomy Observatory, which is operated by the Association of Universities for Research in Astronomy, Inc. under cooperative agreement with the National Science Foundation.
}

available pre-outburst host-galaxy photometry (Melchior et al. 2015; Dong et al. 2016; Leloudas et al. 2016) and have found consistent results. However, the presence of spectral features from the host stellar population has the potential to improve the constraints on the stellar population synthesis, so we used an iterative procedure to incorporate this information while avoiding the flux from the transient.

First, we estimated a best-fit blackbody temperature of $T_{\mathrm{BB}} \approx 15,000 \mathrm{~K}$ at the time of observations from the analysis of the UVOT photometry described above. We then subtracted a scaled blackbody spectrum from the observed spectrum under the constraint that the blackbody-subtracted spectrum had to match the observed colors of the host galaxy to obtain an initial estimate of the host-only spectrum. We then used the FAST code (Kriek et al. 2009) to fit the hostonly spectrum combined with, and normalized by, the broadband grizyJK host photometry (Melchior et al. 2015; Dong et al. 2016). For simplicity, we fixed the metallicity to solar and assumed an initial mass function according to Chabrier (2003) and zero internal extinction. We obtained a satisfactory fit using the stellar models of Bruzual \& Charlot (2003) and an exponentially declining star formation law. The best-fit model has a total stellar mass of $1.2 \times 10^{11} M_{\odot}$, a 
current stellar age of $10 \mathrm{Gyr}$, and an $e$-folding timescale of $2 \mathrm{Gyr}$, resulting in a current star formation rate of $\sim 0.8 M_{\odot} \mathrm{yr}^{-1}$. These numbers are in broad agreement with those reported previously (e.g., Dong et al. 2016; Leloudas et al. 2016). Other choices for the stellar population model produced qualitatively similar results, although usually with smaller current star formation rates. Our best fit for the host is plotted in red in Figure 5.

We then fitted our observed spectrum as a linear combination of the host-galaxy model and a blackbody to find appropriate flux scaling factors. The scaled blackbody is plotted in blue in Figure 5 and good agreement can be seen with the host-subtracted UVOT $u b v$ photometry (green squares) interpolated to the date of observation. Both the fitted host spectrum and the overplotted host photometry (gray circles) have been scaled by a factor of 0.40 from the values for the whole host, which presumably results from the smaller size of our spectroscopic aperture relative to the host as a whole. The models of Bruzual \& Charlot (2003) clearly have narrower features than those visible in our spectrum, so the host template was smoothed with a $10 \AA$ boxcar function to mimic the combined effects of our spectral resolution and the internal velocity dispersion of the host galaxy. Our results are not very sensitive to the width of this smoothing kernel. The sum of the scaled blackbody and the smoothed galaxy template is plotted in magenta in Figure 5 and is a good match to the observed spectrum in black.

Leloudas et al. (2016) noted two emission peaks near 4000 and $5200 \AA$ in their late-time spectra of ASASSN-15lh. However, accurate modeling of the stellar component of the host galaxy from our late-time spectrum demonstrates that the most prominent broad spectral features detected in the observed (host plus transient) late-time spectra have to be attributed to the underlying continuum from the host-galaxy starlight (Figure 5). We do not find unambiguous evidence for broad spectral features associated with the transient at this epoch. Small, broad, low-amplitude discrepancies between the observed spectrum and combined fit (black and magenta lines, respectively) are present, but it is not yet clear whether they represent true spectral features of the transient or limitations in the modeling of stellar population synthesis. More observations of the host will be required after the optical transient fades further to more accurately constrain the presence of possible broad, low-amplitude spectral features in the transient spectrum at late times.

Without any correction for the host galaxy, the spectrum has the two obvious narrow emission features near 8085 and $8111 \AA$ (in air) noted above, which can be clearly associated with $\mathrm{H} \alpha$ and [N II] $\lambda 6583$ at $z=0.2320$ (lower-right panel of Figure 5). ${ }^{17}$ [N II] $\lambda 6548$ is blended in the blue wing of $\mathrm{H} \alpha . \mathrm{H} \beta$ is only visible in emission after subtraction of the host model. Weaker features also appear to be present in the difference spectrum near the [S II] doublet and [O III] $\lambda 5007$. We searched for [O II] $\lambda 3727$ emission and none is visible, but the $\mathrm{S} / \mathrm{N}$ of the spectrum is not as high at those wavelengths. Inspection of our spectral sequence reveals that the $\mathrm{H} \alpha /[\mathrm{N} \mathrm{II}]$ lines are present in several of our higher

\footnotetext{
17 This redshift is consistent with that measured from the stellar absorption features. Note that this value is slightly offset from the redshift $z=0.2326$ measured from narrow UV absorption lines (Brown et al. 2016b; Dong et al. 2016; Leloudas et al. 2016). We do not discuss further the implications of this possible velocity offset for the UV absorbers in this work.
}

$\mathrm{S} / \mathrm{N}$ spectra throughout the evolution of the transient, consistent with a constant low-level contribution that is strongly diluted by light from the transient at earlier times.

The peaks of $\mathrm{H} \alpha$ and [N II] $\lambda 6583$ are of comparable height prior to subtraction of the host model. Strong [N II] $/ \mathrm{H} \alpha$ is a possible sign of ionization by a continuum like that of an active galactic nucleus (AGN). However, after correction for the underlying Balmer absorption in our best-fit host model, the ratio decreases to $\sim 0.5$. This line ratio, combined with weak $[\mathrm{O} \mathrm{III}] / \mathrm{H} \beta$ and $[\mathrm{S} \mathrm{II}] / \mathrm{H} \alpha$, is consistent with the nebular emission being powered by star formation instead of AGN activity (Kewley et al. 2006). We caution that these ratios are sensitive to systematic errors in the modeling of the underlying stellar absorption, and in particular the strength of the stellar Balmer absorption. If all of the inferred $\mathrm{H} \alpha$ emission (flux $\sim 2.7 \times 10^{-16} \mathrm{erg} \mathrm{cm}^{-2} \mathrm{~s}^{-1}$ ) is powered by star formation, the inferred rate is $\sim 0.4 M_{\odot} \mathrm{yr}^{-1}$ (Kennicutt 1998), in rough agreement with that estimated from the fit to the stellar population of the host galaxy.

Leloudas et al. (2016) reported $\mathrm{H} \alpha$ emission from ASASSN-15lh with an FWHM of $2500 \mathrm{~km} \mathrm{~s}^{-1}$, but in our data it is clear that the reported emission feature is just the narrow nebular $\mathrm{H} \alpha$ and [N II] from the host blended together at low $\mathrm{S} / \mathrm{N}$ or low resolution in their data. Note that in their highest $\mathrm{S} / \mathrm{N}$ spectra (inset of their Figure 1), the putative $\mathrm{H} \alpha$ from the transient is flat-topped or double-peaked, consistent with the two strong nebular emission lines of roughly equal height (inset of our Figure 5) being blended together. We also note that Leloudas et al. (2016) do not attempt to correct for the contribution from the underlying stellar continuum. Therefore, we do not confirm their claim of $\mathrm{H} \alpha$ emission from the transient itself and the reported velocity FWHM likely reflects the spacing of the two [N II] lines, which are each offset by $\sim 1000 \mathrm{~km} \mathrm{~s}^{-1}$ from the central $\mathrm{H} \alpha$ emission. Godoy-Rivera et al. (2016) also report a "bump" near $\mathrm{H} \alpha$ at late times, but they do not report an FWHM, so it is not clear whether they are also possibly referring to a noisy detection of the narrow nebular lines.

\subsection{Reanalysis of Early-time Optical Spectra}

In addition, we re-evaluated the early optical spectra of ASASSN-15lh and were unable to confirm the likeness to SLSNe reported by Dong et al. (2016). The O II ion, which is commonly observed in SLSNe (Quimby et al. 2011), has a number of distinctive absorption features not observed in ASASSN-15lh (Figure 6). The strongest two features centered near 4100 and $4400 \AA$ are always observed to be of comparable strength and no reasonable values of temperature or density can change this ratio. ASASSN-15lh shows only the $4100 \AA$ feature (Figure 6, see also Leloudas et al. 2016). Without the accompanying $4400 \AA$ feature, it is hard to reconcile the proposed association with $\mathrm{O}$ II, and thus the spectroscopic connection to SLSNe is not robust.

The spectral features of ASASSN-15lh trend redward over time toward declining velocities. This is similar to the spectral evolution of supernovae where the trend is attributed to an expanding and cooling photosphere. However, unlike supernovae, the features of ASASSN-15lh do not show traditional P-Cyg profiles and become increasingly inconspicuous. For example, the +30 day spectrum of SN 2010gx exhibits pronounced features, whereas the +39 day ASASSN-15lh 


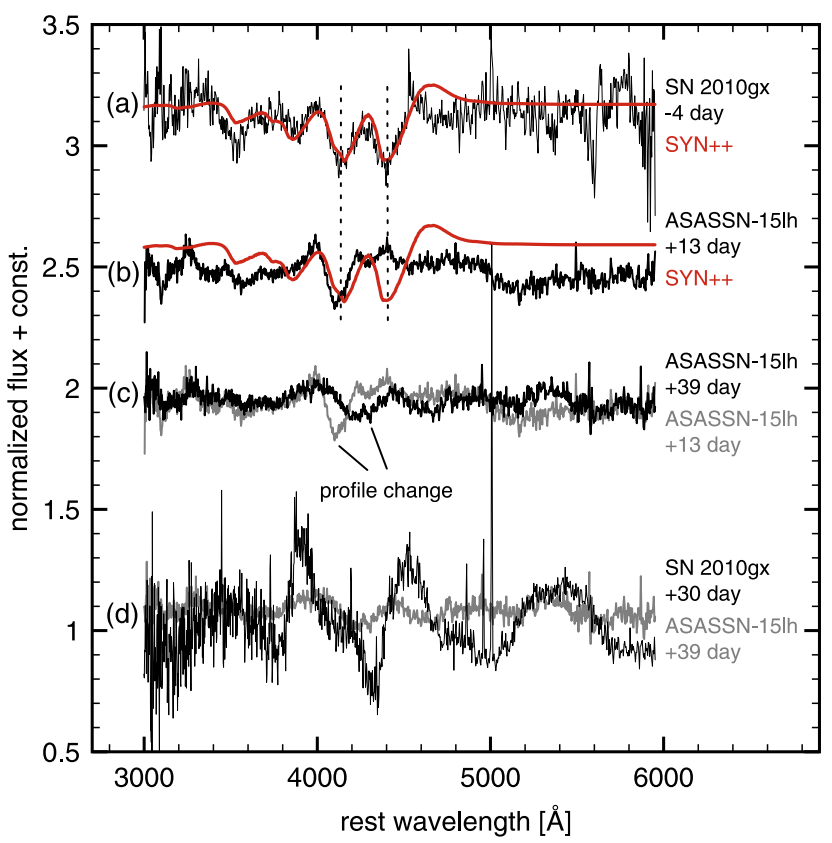

Figure 6. Placing early-phase optical spectra of ASASSN-15lh in context with SLSNe. (a) The O II ion is a signature of SLSNe, and here we show how the -4 day spectrum of the SLSN 2010gx can be reproduced using the simple assumptions of SYN++ and a photospheric velocity of $19,000 \mathrm{~km} \mathrm{~s}^{-1}$. (b) By contrast, we cannot reproduce +13 day spectrum of ASASSN-15lh. It clearly misses an accompanying feature around $4400 \AA$. (c) Evolution in the spectra is observed. Most conspicuous is the $4100 \AA$ feature, which drifts to longer wavelengths. (d) The evolution toward increasingly inconspicuous spectral features is unlike SLSNe that exhibit increasingly stronger spectral features. Here we show the +30 day spectrum of SN 2010gx, which is unlike the nearly featureless +39 day spectrum of ASASSN-15lh. Data have been retrieved from WISEREP (Yaron \& Gal-Yam 2012), normalized according to the procedure outlined in Jeffery et al. (2007) to aid in visual comparison, and were originally published in Pastorello et al. (2010) and Dong et al. (2016).

spectrum is nearly featureless (Figure 6). We explored a variety of possible ions using the highly parameterized spectrum synthesis tool, SYN++ (Thomas et al. 2011), to determine whether blending of features could reproduce the spectral features and evolution of ASASSN-15lh, but were unsuccessful.

To our knowledge the only previous examples of spectral features becoming increasingly inconspicuous in the early phases of a supernova involve interaction with dense CSM. $\mathrm{SN}-\mathrm{CSM}$ interaction can rescale or "mute" the line profile relative to the continuum (Branch et al. 2000). Most confirmed instances of SN-CSM interaction involve H-rich material that can be readily identified by the presence of $\mathrm{H}$ Balmer lines that may be narrow $\left(<100 \mathrm{~km} \mathrm{~s}^{-1}\right)$ to broad $\left(\sim 10^{3} \mathrm{~km} \mathrm{~s}^{-1}\right)$ in width, depending on their origin of formation. The hydrogenpoor SLSN iPTF13ehe exhibited $\mathrm{H} \alpha$ Balmer emission with broad and narrow components +251 days after maximum (Yan et al. 2015). However, no such lines are observed in ASASN$15 \mathrm{lh}$. Interaction with $\mathrm{H}$-poor CSM ejected by rapidly rotating pulsational pair instability supernovae is possible (Chatzopoulos \& Wheeler 2012; Chatzopoulos et al. 2016), but the spectroscopic consequences of such interaction are poorly understood (Chatzopoulos et al. 2013), and the timescales of variability observed in the UV strongly disfavor this scenario (Section 2.3).
A luminous central source overionizing expanding ejecta is a speculative, though attractive, scenario that may explain the spectroscopic evolution of ASASSN-15lh toward a featureless continuum. As there is no precedent for this scenario, the specific spectral signatures are unclear. Certainly, the ionizing photons must be extremely energetic for no strong optical or UV lines to be observable. An analogous phenomenon may be variable UV absorption commonly seen in Seyfert galaxies (Maran et al. 1996; Crenshaw et al. 2000). In some cases variability in the form of absorption components appearing and disappearing, or decreasing outflow velocities (Gabel et al. 2003), can result from changes in the ionizing flux (Kraemer et al. 2002). ASASSN-15lh may be an extreme version of these processes.

\section{Interpretation}

\subsection{The "Reprocessing Picture"}

Although the mechanisms behind SLSNe powered by a stellar-mass compact object-such as a magnetar-and the tidal disruption and accretion of a star by an SMBH do differ significantly, the basic physical process driving the light curves of these events may be similar. A central source of UV/X-ray radiation (an accreting $\mathrm{SMBH}$ or the pulsar wind nebula of a rapidly spinning neutron star, NS) is absorbed by a dense column of gas, and downgraded into optical radiation, where the lower opacity allows the radiation to more readily escape. Such a "reprocessing" picture has been applied to explain both TDEs (Loeb \& Ulmer 1997; Guillochon \& Ramirez-Ruiz 2013; Metzger \& Stone 2016) and SLSNe (Kasen \& Bildsten 2010; Woosley 2010; Metzger et al. 2014).

Consider the characteristic timescale of the central engine in a magnetar-powered SLSN and in a TDE scenario. In a magnetar-powered SLSN, the lifetime of the central engine is the magnetic dipole spin-down timescale of the magnetar:

$$
t_{\mathrm{sd}} \simeq 17 \text { days }\left(M / 1.4 M_{\odot}\right)^{3 / 2} B_{13}^{-2} P_{\mathrm{ms}}^{2},
$$

where $M, P=P_{\mathrm{ms}} \mathrm{ms}$, and $B_{\mathrm{days}}=10^{13} B_{13} \mathrm{G}$ are, respectively, the mass, initial spin period, and dipole surface magnetic field strength of the magnetar (e.g., Spitkovsky 2006). The maximum energy of the engine is limited to the rotational energy of the NS,

$$
E_{\mathrm{rot}}=I \Omega^{2} / 2 \simeq 2.5 \times 10^{52} \mathrm{erg}\left(M / 1.4 M_{\odot}\right)^{3 / 2} P_{\mathrm{ms}}^{-2},
$$

which can vary from $\sim 3 \times 10^{52}$ to $10^{53}$ erg for the minimum value of the spin period set by the mass-shedding limit, depending on the mass and equation of state of the NS (Metzger et al. 2015). In order to simultaneously explain the large radiated energy and duration of ASASSN-15lh with a magnetar, we require a maximally spinning neutron star $\left(P_{\mathrm{ms}} \lesssim 1\right)$ and a relatively weak magnetic field $B_{\text {days }} \sim 10^{12}$ $10^{13} \mathrm{G}$ (e.g., Metzger et al. 2015; see also Bersten et al. 2016; Chatzopoulos et al. 2016; Dong et al. 2016; Sukhbold \& Woosley 2016).

In the TDE scenario, the lifetime of the engine is uncertain, but is commonly attributed to the fall-back time of the most tightly bound stellar debris following the disruption (e.g., 
Guillochon \& Ramirez-Ruiz 2013; Stone et al. 2013),

$$
t_{\mathrm{fb}} \approx 410 \text { days }\left(\frac{M .}{10^{8} M_{\odot}}\right)^{1 / 2}\left(\frac{m_{\star}}{M_{\odot}}\right)^{1 / 5},
$$

where $M$. and $m_{\star}$ are the masses of the SMBH and the star, respectively, and we have assumed a stellar mass-radius relationship $R_{\star} \simeq R_{\odot}\left(m_{\star} / M_{\odot}\right)^{4 / 5}$ appropriate to lower mainsequence stars. The maximum radiated energy is that liberated by the accretion of the half of the stellar mass that remains bound to the SMBH,

$$
E_{\text {acc }}=\eta\left(m_{\star} / 2\right) c^{2} \simeq 9 \times 10^{52} \operatorname{erg}\left(\frac{\eta}{0.1}\right)\left(\frac{m_{\star}}{M_{\odot}}\right),
$$

where the radiative efficiency for geometrically thin accretion varies from $\eta \approx 0.04$ to 0.42 , depending on the spin of the SMBH and its orientation relative to the angular momentum of the accreting gas.

In the TDE scenario, the energetics of ASASSN-15lh are reasonably accommodated by the accretion of a solar-mass star. However, the high-mass SMBH $M_{\bullet} \sim 6 \times 10^{8} M_{\odot}$ inferred from the host of ASASSN-15lh is inconsistent with the tidal disruption of a solar-mass star by a Schwarzschild SMBH because the tidal radius is smaller than the innermost stable stellar orbit for $M_{\bullet} \gtrsim 10^{7.4} M_{\odot}$ (i.e., the star would be swallowed whole instead of producing a bright flare). This discrepancy could be alleviated if the SMBH is spinning in a prograde direction with respect to the orbit of the disrupted star, in which case tidal disruption is possible even for more massive BHs (e.g., Kesden 2012).

The high inferred SMBH mass would also appear to predict a long duration of the transient $t_{\mathrm{fb}} \gtrsim 2 \mathrm{yr}$, inconsistent with the much shorter observed decay time of the first peak of a few weeks. This inconsistency might also be resolved by a prograde spinning SMBH. Precession of the star during the phase of tidal compression due to the $\mathrm{BH}$ spin may substantially enhance the spread in the energy distribution of the stellar debris as compared to the Newtonian case, by partially aligning the direction of the hydrodynamic bounce with the velocity vector of the star (Stone et al. 2013; Leloudas et al. 2016; Metzger \& Stone 2016). More tightly bound debris has a shorter orbital period, which could significantly speed up the timescale of flare evolution as compared to the estimate from Newtonian gravity in Equation (3). Though it is promising, generalrelativistic numerical simulations are needed to confirm this possibility.

In addition to possibly speeding up the flare evolution, the high BH spin required to explain ASASSN-15lh as a TDE would (i) naturally result in a large value of the accretion efficiency $\eta$, accounting for its high luminosity, and (ii) possibly aid in the process of debris circularization by allowing relativistic pericenters, thus inducing greater general-relativistic precession of the stellar debris streams (e.g., Dai et al. 2015; Bonnerot et al. 2016; Hayasaki et al. 2016). Precession of the streams out of the orbital plane due to misaligned $\mathrm{BH}$ spin could also help make the geometry of the reprocessing material relatively spherical (e.g., Guillochon \& Ramirez-Ruiz 2015; Hayasaki et al. 2016), consistent with the low measured optical polarization of ASASSN-15lh reported by Brown et al. (2016b). We end by noting that the lack of conspicuous $\mathrm{H}$ emission from ASASSN-15lh cannot be used as an argument against the tidal disruption of a star of solar composition. As shown by Roth et al. (2016), H emission lines can be suppressed below the continuum level, depending on the precise conditions in the reprocessing layer.

\subsection{Escape of X-Rays and the Ionization Break-out}

In both the magnetar SLSN and the TDE scenario, UV/Xray radiation from the central source may ionize its way through the ejecta at late times. This process can result in the direct escape of UV/soft X-ray radiation while having an indirect influence on the observed optical light curve by changing the ejecta opacity (Section 3.3).

If we approximate the ejecta as a homogeneously expanding sphere of mass $M_{\mathrm{ej}}$, velocity $v_{\mathrm{ej}}=10^{9} v_{9} \mathrm{~cm} \mathrm{~s}^{-1}$, and radius $R_{\mathrm{ej}}=v_{\mathrm{ej}} t$, then the neutral column density is

$$
\begin{aligned}
\Sigma(t) & \simeq \frac{M_{\mathrm{ej}}}{(4 \pi / 3) R_{\mathrm{ej}}^{2} m_{p}} \\
& \approx 1.7 \times 10^{24} \mathrm{~cm}^{-2} f_{n}\left(\frac{M_{\mathrm{ej}}}{M_{\odot}}\right) v_{9}^{-2}\left(\frac{t}{150 \text { days }}\right)^{-2},
\end{aligned}
$$

where $f_{n}$ is the neutral fraction. This is much higher than the inferred X-ray absorption column of $\mathrm{NH}_{\text {int }}<3 \times 10^{22} \mathrm{~cm}^{-2}$ toward ASASSN-15lh, requiring a very low neutral fraction if the X-ray source is related to the optical transient. This is consistent with the very low $N(\mathrm{H} \mathrm{I})$ inferred by Leloudas et al. (2016) from $\mathrm{Ly} \alpha$.

The ejecta from TDEs and SLSNe are expected to have markedly different chemical composition. In a TDE the ejecta has nearly solar composition (e.g., Kochanek 2016) and the escape of soft X-rays is inhibited primarily by the bound-free opacity of neutral helium (Metzger \& Stone 2016; Roth et al. 2016). By contrast, in an H-poor SLSN, X-rays are blocked more severely by neutral oxygen and carbon (Metzger et al. 2014).

A central engine with a UV/X-ray luminosity $L$ releases an energy $L \times t$ in ionizing radiation on a timescale $t$. If the ejecta contains a mass fraction $X_{Z}$ of elements with atomic number $Z=8 Z_{8}$, then the radiation ionizes its way through the ejecta on a timescale

$$
t_{\text {ion }} \approx\left\{\begin{array}{l}
120 \text { days } M_{3}^{3 / 4} v_{9}^{-5 / 4} T_{5}^{-0.2}\left(\frac{X_{A}}{0.1}\right)^{1 / 4}\left(\frac{L t}{10^{52} \mathrm{erg}}\right)^{-1 / 4} Z_{8}^{3 / 4}, \\
\left(\eta_{\mathrm{thr}} \ll 1\right) \\
110 \text { days } M_{3} v_{9}^{-3 / 2} T_{5}^{-0.4}\left(\frac{X_{A}}{0.1}\right)^{1 / 2}\left(\frac{L t}{10^{52} \mathrm{erg}}\right)^{-1 / 2} Z_{8}^{3 / 2}, \\
\left(\eta_{\mathrm{thr}} \gg 1\right),
\end{array}\right.
$$

where $M_{3} \equiv M_{\mathrm{ej}} /\left(3 M_{\odot}\right), T_{5}=T / 10^{5} \mathrm{~K}$ is the temperature of electrons in the recombination layer, and

$$
\eta_{\mathrm{thr}} \approx 0.7\left(\frac{L t}{10^{52} \mathrm{erg}}\right)^{-1} M_{3} v_{9}^{-1}\left(\frac{X_{A}}{0.1}\right) T_{5}^{-0.8} Z_{8}^{3}
$$

is the ratio of absorptive and scattering opacity in the ejecta (Metzger et al. 2014). 
For typical parameters and an engine similar to ASASSN$15 \mathrm{lh}$ with $L t \sim 10^{52} \mathrm{erg}$, we have $t_{\text {ion }} \sim 1$ month in the case of a He-rich composition $(Z=2)$ of a TDE-like scenario. By contrast, for a CO-rich composition of an exploding massive star $(Z=8)$, we have $t_{\text {ion }} \sim$ several months, making break-out harder to achieve. In the latter case, X-ray break-out is even less likely considering that the $\mathrm{K}$-shell valence electrons of oxygen have a binding energy of $\sim 1 \mathrm{keV}$, while the measured $\mathrm{keV}$ X-ray luminosity of ASASSN-15lh, $\leqslant 10^{42} \mathrm{erg} \mathrm{s}^{-1}$, is much less than the optical/UV luminosity (in other words, the true value of $L t$ to use in Equation (6) should be much lower than $10^{52} \mathrm{erg}$ ).

We conclude that an ionization break-out could allow the escape of X-rays in the TDE scenario, but is probably not sufficient to do so in the case of an H-poor supernova given the observed soft X-ray spectrum.

\subsection{The Double-humped Light Curve of ASASSN-15lh}

The ionization of the ejecta reduces the bound-free opacity, allowing the escape to the observer of UV and X-ray radiation with energies above the ionization threshold. This process is unlikely to explain the observed UV rebrightening by itself, because even the highest-frequency UV bands of Swift-UVOT are below the first ionization energies of the most abundant elements $(\mathrm{H}, \mathrm{He}, \mathrm{C}, \mathrm{O})$. However, an ionization break-out may have an indirect effect on the light curve via the continuum opacity.

At early times the ejecta is largely neutral and the opacity at optical frequencies is dominated by electron scattering, while the opacity at UV frequencies is dominated by line transitions of metals. However, once the ejecta becomes ionized by the central engine, the electron scattering opacity will increase, while the UV opacity will decrease as the ionized atoms have fewer bound-bound transitions. Therefore, following ionization break-out we expect a shift of the peak of the spectral energy distribution from optical to UV frequencies. It is unclear at the moment whether this effect would be able to quantitatively explain the slow decay of the optical emission after the break-out observed in ASASSN-15lh (e.g., GodoyRivera et al. 2016, their Figure 1). Here we note that, according to Roth et al. (2016), the continuum receives significant contributions from free-free emission, in which case the higher temperature of the ejecta caused by the ionization break-out might enhance the optical luminosity. Detailed radiative transfer calculations are necessary to quantitatively address this issue, and will be performed elsewhere. The appeal of this model is that a single timescale for the central engine would naturally reproduce the doublepeaked temporal structure of ASASSN-15lh, which has no analogue in previously observed TDE or SLSN light curves. As a comparison, the TDE model invoked by Leloudas et al. (2016) combines two luminosity mechanisms, which result in two different timescales.

While we emphasize that accurate modeling, beyond the scope of this paper, is necessary to understand whether this effect alone can quantitatively explain the observations of ASASSN-15lh, here we consider a toy model to illustrate the basic principles. For illustrative purposes we use the spin-down luminosity of a magnetar as the central source of ionizing photons. In particular, we consider a magnetar light curve with parameters $P=1 \mathrm{~ms}$ and $B_{\text {days }}=3 \times 10^{12} \mathrm{G}$, similar to that

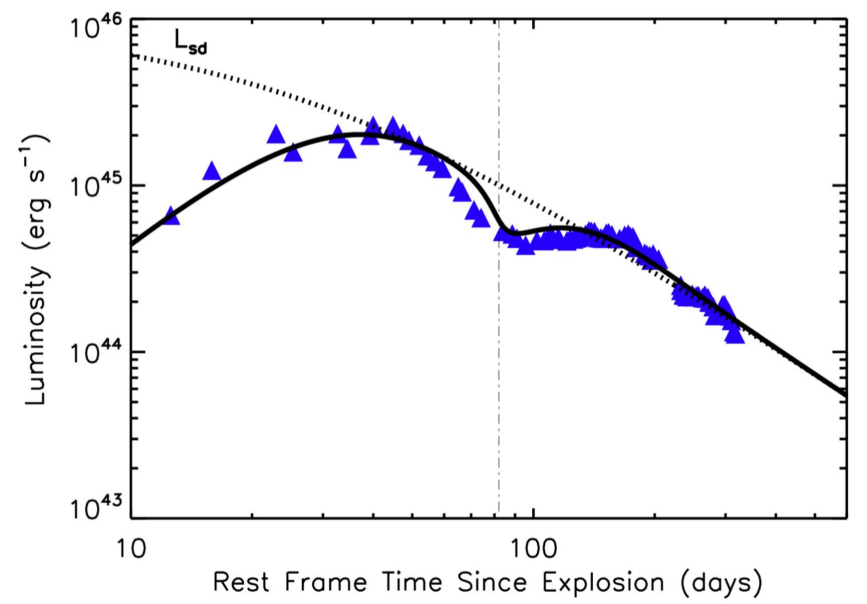

Figure 7. Bolometric model of the light curve (solid line) due to reprocessed luminosity from a central source (dashed line). For this plot we use the spindown power from a magnetar engine as central source as an example. We artificially and abruptly change the ejecta opacity at the time shown by the vertical dashed line to approximate the effect of an ionization break-out. The object's time of first light is assumed to occur 30 days before the peak of the light curve. The bolometric light curve of ASASSN-15lh that we derived in Section 2.3 is shown for comparison with blue triangles. Accurate modeling is necessary to quantitatively understand whether the change in opacity alone can be responsible for the observed phenomenology.

described in Metzger et al. (2015), and a total ejecta mass of $M_{\mathrm{ej}}=10 M_{\odot}$. However, we artificially change the gray opacity from $\kappa_{i}=0.02 \mathrm{~cm}^{2} \mathrm{~g}^{-1}$ to $\kappa_{f}=0.2 \mathrm{~cm}^{2} \mathrm{~g}^{-1}$ at a time corresponding to ionization break-out of about 50 days. As shown in Figure 7, this produces a minimum/flattening in the bolometric light curve, similar to that observed in ASASSN15lh. Although we have applied the model to a magnetar for concreteness (and since the process of debris circularization in TDEs remains uncertain), a similar result applies to the TDE case if the central UV/X-ray accretion power rises smoothly on a timescale of a few weeks and then decays $\propto t^{-5 / 3}$ at later times. We also caution that a simple change in the gray opacity is unlikely to accurately predict the effect of a wavelengthdependent change in opacity created by an ionization breakout. Finally we note that in this model the late-time UV variability (Section 2.3, Figure 4) has to be ascribed to the central engine, because the reduced opacity after ionization break-out would allow the observed light curve to more faithfully track the luminosity of the central source (i.e., $t_{\text {diff }} \ll t_{\text {obs }}$ ).

\section{Summary and Conclusions}

We have presented evidence for luminous, soft, and persistent X-ray emission at the location of ASASSN-15lh, and discussed its origin in the context of multiwavelength observations of the transient, which include constraints on its radio emission and early- and late-time optical spectroscopy. Our reanalysis of early-time spectra does not confirm the robust association of ASASSN-15lh with SLSNe claimed by previous studies, and invites us to be open-minded about the nature of ASASSN-15lh. Late-time spectra reveal the emergence of narrow emission features from the host galaxy, while we associate the most prominent broad spectral features with the underlying stellar population. No clear evidence is found for 
broad spectral features associated with the transient at late times.

We propose a model that explains the double-peaked temporal structure of ASASSN-15lh in the optical/UV band as originating from the temporal evolution of the ejecta opacity, which changes as a result of persistent ionizing flux from a long-lived central source (either a magnetar or an accreting $\mathrm{SMBH}$ ). Detailed radiative transfer simulations are necessary to quantitatively compare our idea to the observations. We speculate that the evolution of ASASSN-15lh toward a featureless spectrum also results from the presence of a persistent central source of ionizing photons. The exceptionally long active timescale and high luminosity of the ionizing central source powering ASASSN-15lh (i.e., months) is most likely the key physical property that distinguishes ASASSN-15lh from all the TDEs and SLSNe discovered so far.

The optical/UV spectral evolution of ASASSN-15lh, its peculiar rebrightening, and the presence of soft and persistent X-ray emission are indeed unprecedented among SLSNe and TDEs and suggest two scenarios: (i) either ASASSN-15lh is the first member of a class of stellar explosions with extreme properties that are intrinsically rare or that have been overlooked because of their location very close to the host-galaxy nucleus or, alternatively, (ii) ASASSN-15lh results from refreshed nuclear activity of the host-galaxy SMBH.

In the first scenario the detected X-ray emission is physically unrelated to the transient and most likely originates from the host-galaxy nucleus. We thus expect no fading of the X-ray source over the timescales of years.

Instead, if the X-ray emission is physically associated with the optical/UV transient, then ASASSN-15lh is unlikely to originate from a stellar explosion, and an association with the activity of the host nucleus is favored. In this case, ASASSN$15 \mathrm{lh}$ would be a TDE from the most massive spinning SMBH observed to date. The fast initial decay timescale of the transient is challenging to understand based on the fall-back timescale of the disrupted star in Newtonian gravity, possibly suggesting that $\mathrm{BH}$ spin plays a key role in enhancing the energy spread of the disrupted star. ASASSN-15lh and similar events discovered in the future would then constitute direct probes of matter under strong gravity around very massive, dormant, spinning SMBHs in galaxies. We emphasize that this scenario predicts significant temporal evolution of the X-ray emission over the next few years, because we expect a TDE to have a non-negligible impact on the inner part of the accretion disk even in the case of a pre-existing weak AGN.

Continued deep X-ray monitoring of ASASSN-15lh will constrain the temporal evolution of the X-ray source and its fading, revealing in this way whether the X-ray source is indeed physically related to the optical/UV transient. Future X-ray observations thus hold the keys to unveiling the true nature of ASASSN-15lh.

We thank the referee for constructive criticism and suggestions that improved the quality of this work. R.M. acknowledges partial support from the James Arthur Fellowship at NYU during the completion of this project and the Research Corporation for Science Advancement. B.D.M. gratefully acknowledges support from the NSF (AST1410950, AST-1615084), NASA Astrophysics Theory Program (NNX16AB30G), the Alfred P. Sloan Foundation, and the Research Corporation for Science Advancement. G.M. acknowledges the financial support from the UnivEarthS Labex program of Sorbonne Paris Cité (ANR10LABX0023 and ANR11IDEX000502). The scientific results reported in this article are based on observations made by the Chandra $X$-ray Observatory under program GO 17500103, PI Margutti, observations IDs 17879, 17880, 17881, 17882. This paper includes data gathered with the 6.5 meter Magellan Telescopes located at Las Campanas Observatory, Chile.

Facility: Magellan:Clay.

\section{Appendix \\ X-ray and UV/Optical Photometry Tables}

Tables 1 and 2 show a summary of results from X-ray and photometry observations.

Table 1

X-ray Observations

\begin{tabular}{|c|c|c|c|c|}
\hline Date (MJD) & Instrument & Exposure (ks) & Unabsorbed Flux (erg s${ }^{-1} \mathrm{~cm}^{-2}$ ) & Spectral Model \\
\hline \multirow[t]{2}{*}{$57046-57591$} & Swift/XRT & 270 & $F_{x}=(4.1 \pm 1.5) \times 10^{-15}$ & $\mathrm{BB}$ \\
\hline & & & $F_{x}=(3.7 \pm 1.4) \times 10^{-15}$ & PL \\
\hline \multirow[t]{2}{*}{57338} & $\mathrm{CXO}$ & 10 & $F_{x}<2.0 \times 10^{-15}$ & PL \\
\hline & & & $F_{x}<8.0 \times 10^{-16}$ & BB \\
\hline \multirow[t]{2}{*}{57344} & $X M M$ & 9 & $F_{x}<1.5 \times 10^{-14}$ & PL \\
\hline & & & $F_{x}<1.4 \times 10^{-14}$ & $\mathrm{BB}$ \\
\hline \multirow[t]{2}{*}{57369} & $C X O$ & 10 & $F_{x} \sim 4.4 \times 10^{-15}$ & PL \\
\hline & & & $F_{x} \sim 1.6 \times 10^{-15}$ & BB \\
\hline \multirow[t]{2}{*}{57438} & $\mathrm{CXO}$ & 40 & $F_{x} \sim 3.6 \times 10^{-15}$ & PL \\
\hline & & & $F_{x} \sim 1.2 \times 10^{-15}$ & BB \\
\hline \multirow[t]{2}{*}{57619} & $\mathrm{CXO}$ & 30 & $F_{x} \sim 4.9 \times 10^{-15}$ & PL \\
\hline & & & $F_{x} \sim 1.4 \times 10^{-15}$ & $\mathrm{BB}$ \\
\hline
\end{tabular}

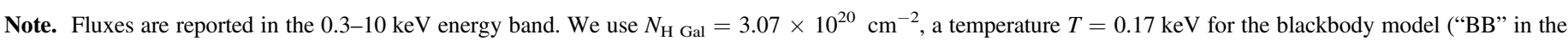

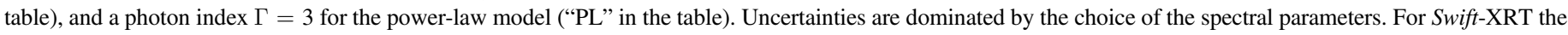
reported uncertainties reflect the count-rate statistics only. 
Table 2

Swift-UVOT Photometry

\begin{tabular}{|c|c|c|c|c|c|c|c|c|c|c|c|}
\hline Date & $v(\mathrm{mag})$ & Date & $b$ (mag) & Date & $u$ (mag) & Date & $w 1$ (mag) & Date & $w 2(\mathrm{mag})$ & Date & $m 2$ (mag) \\
\hline $197.10^{\mathrm{a}}$ & $16.86(0.07)$ & 197.09 & $16.76(0.04)$ & 197.09 & $15.39(0.04)$ & 197.09 & $15.27(0.04)$ & 197.10 & $15.63(0.04)$ & 197.10 & $15.25(0.04)$ \\
\hline 199.79 & $16.85(0.07)$ & 199.79 & $16.82(0.04)$ & 199.79 & $15.43(0.04)$ & 199.79 & $15.33(0.04)$ & 199.79 & $15.67(0.04)$ & 199.79 & $15.35(0.04)$ \\
\hline 201.82 & $16.93(0.08)$ & 201.82 & $16.87(0.04)$ & 201.82 & $15.48(0.04)$ & 201.82 & $15.40(0.04)$ & 201.82 & $15.83(0.04)$ & 201.82 & $15.48(0.05)$ \\
\hline 205.64 & $16.98(0.08)$ & 205.64 & $16.94(0.05)$ & 205.64 & $15.54(0.04)$ & 205.63 & $15.56(0.04)$ & 205.64 & $15.88(0.04)$ & 205.64 & $15.58(0.04)$ \\
\hline 208.66 & $17.05(0.08)$ & 208.66 & $17.03(0.05)$ & 208.66 & $15.72(0.04)$ & 208.66 & $15.74(0.04)$ & 208.66 & $16.07(0.04)$ & 208.67 & $15.76(0.04)$ \\
\hline 211.66 & $17.07(0.09)$ & 211.66 & $17.05(0.05)$ & 211.66 & $15.77(0.04)$ & 211.66 & $15.83(0.05)$ & 211.66 & $16.18(0.05)$ & 211.66 & $15.94(0.04)$ \\
\hline 221.69 & $17.27(0.13)$ & 214.68 & $17.09(0.05)$ & 214.68 & $15.85(0.04)$ & 214.67 & $15.95(0.05)$ & 214.68 & $16.35(0.04)$ & 214.68 & $16.06(0.05)$ \\
\hline 223.52 & $17.32(0.10)$ & 221.68 & $17.29(0.07)$ & 216.56 & $15.90(0.03)$ & 219.79 & $16.26(0.06)$ & 217.01 & $16.48(0.04)$ & 221.69 & $16.52(0.05)$ \\
\hline 231.07 & $17.51(0.08)$ & 223.51 & $17.27(0.06)$ & 221.68 & $16.03(0.06)$ & 221.68 & $16.31(0.06)$ & 220.69 & $16.71(0.05)$ & 223.52 & $16.68(0.05)$ \\
\hline 244.89 & $17.54(0.11)$ & 229.27 & $17.41(0.06)$ & 220.52 & $16.08(0.04)$ & 223.51 & $16.45(0.05)$ & 223.51 & $16.90(0.05)$ & 229.27 & $16.99(0.05)$ \\
\hline 255.12 & $17.58(0.07)$ & 232.89 & $17.45(0.06)$ & 223.51 & $16.18(0.05)$ & 229.27 & $16.74(0.06)$ & 229.27 & $17.25(0.06)$ & 232.90 & $17.26(0.06)$ \\
\hline 268.79 & $17.59(0.12)$ & 244.88 & $17.71(0.07)$ & 229.27 & $16.39(0.05)$ & 232.89 & $16.99(0.06)$ & 232.89 & $17.50(0.06)$ & 244.89 & $17.70(0.06)$ \\
\hline 310.35 & $17.80(0.08)$ & 250.70 & $17.49(0.09)$ & 232.89 & $16.48(0.05)$ & 244.88 & $17.43(0.07)$ & 244.88 & $17.99(0.07)$ & 250.71 & $17.81(0.10)$ \\
\hline 383.29 & $18.18(0.32)$ & 253.70 & $17.66(0.10)$ & 244.88 & $16.76(0.06)$ & 250.70 & $17.31(0.10)$ & 250.71 & $18.07(0.11)$ & 253.63 & $17.76(0.13)$ \\
\hline 214.68 & $17.16(0.09)$ & 259.40 & $17.87(0.08)$ & 250.70 & $16.83(0.09)$ & 253.67 & $17.43(0.08)$ & 253.70 & $18.11(0.12)$ & 259.41 & $18.09(0.08)$ \\
\hline 226.52 & $17.39(0.07)$ & 265.56 & $17.82(0.07)$ & 253.70 & $16.68(0.08)$ & 259.40 & $17.55(0.08)$ & 259.40 & $18.46(0.10)$ & 268.79 & $18.08(0.09)$ \\
\hline 241.13 & $17.57(0.09)$ & 268.79 & $17.89(0.08)$ & 259.40 & $16.97(0.07)$ & 265.56 & $17.51(0.07)$ & 267.22 & $18.29(0.08)$ & 283.28 & $17.45(0.06)$ \\
\hline 248.47 & $17.51(0.09)$ & 309.34 & $18.06(0.06)$ & 265.56 & $17.05(0.06)$ & 268.78 & $17.51(0.07)$ & 283.88 & $17.82(0.04)$ & 290.87 & $17.37(0.06)$ \\
\hline 262.73 & $17.67(0.10)$ & 313.24 & $17.95(0.09)$ & 268.79 & $17.00(0.07)$ & 282.88 & $17.18(0.06)$ & 290.88 & $17.71(0.06)$ & 293.56 & $17.03(0.06)$ \\
\hline 272.67 & $17.68(0.10)$ & 383.28 & $18.59(0.16)$ & 282.88 & $16.89(0.05)$ & 283.41 & $17.14(0.06)$ & 293.57 & $17.56(0.06)$ & 296.62 & $17.06(0.06)$ \\
\hline 277.36 & $17.55(0.07)$ & 226.52 & $17.35(0.04)$ & 283.41 & $16.93(0.05)$ & 290.87 & $17.16(0.06)$ & 296.63 & $17.50(0.05)$ & 299.51 & $17.14(0.06)$ \\
\hline 284.57 & $17.77(0.07)$ & 241.12 & $17.58(0.05)$ & 290.87 & $16.93(0.05)$ & 293.56 & $17.03(0.06)$ & 299.52 & $17.51(0.05)$ & 302.57 & $17.06(0.06)$ \\
\hline 298.65 & $17.84(0.11)$ & 248.47 & $17.60(0.05)$ & 293.56 & $16.99(0.05)$ & 296.62 & $16.90(0.05)$ & 302.57 & $17.55(0.05)$ & 307.46 & $16.97(0.05)$ \\
\hline 305.49 & $17.90(0.10)$ & 262.73 & $17.88(0.06)$ & 296.62 & $17.02(0.05)$ & 299.51 & $16.96(0.06)$ & 307.45 & $17.28(0.06)$ & 309.68 & $16.95(0.06)$ \\
\hline 317.77 & $17.93(0.14)$ & 272.67 & $17.83(0.05)$ & 299.52 & $16.96(0.05)$ & 302.57 & $17.00(0.05)$ & 309.68 & $17.30(0.07)$ & 311.31 & $16.81(0.05)$ \\
\hline 330.19 & $17.93(0.11)$ & 277.36 & $17.72(0.05)$ & 302.57 & $16.90(0.05)$ & 307.45 & $16.93(0.06)$ & 311.31 & $17.20(0.06)$ & 313.24 & $16.93(0.05)$ \\
\hline 346.20 & $17.92(0.10)$ & 284.57 & $17.86(0.05)$ & 307.45 & $16.91(0.06)$ & 309.67 & $16.81(0.07)$ & 313.24 & $17.27(0.06)$ & 353.56 & $16.96(0.07)$ \\
\hline 371.42 & $18.19(0.17)$ & 298.64 & $18.09(0.07)$ & 309.68 & $16.84(0.08)$ & 311.30 & $16.85(0.06)$ & 353.63 & $17.30(0.10)$ & 356.22 & $17.12(0.07)$ \\
\hline 393.66 & $18.10(0.15)$ & 255.69 & $17.74(0.05)$ & 311.30 & $16.92(0.07)$ & 313.23 & $16.83(0.06)$ & 356.23 & $17.21(0.05)$ & 359.31 & $17.04(0.07)$ \\
\hline 447.41 & $18.49(0.29)$ & 305.49 & $17.98(0.06)$ & 313.23 & $16.93(0.07)$ & 353.56 & $16.91(0.07)$ & 359.32 & $17.29(0.06)$ & 362.84 & $17.21(0.08)$ \\
\hline 398.03 & $18.02(0.11)$ & 317.77 & $17.91(0.07)$ & 353.57 & $16.94(0.09)$ & 356.23 & $16.96(0.06)$ & 362.84 & $17.23(0.06)$ & 365.09 & $17.19(0.07)$ \\
\hline 432.60 & $18.59(0.19)$ & 330.18 & $18.08(0.08)$ & 356.23 & $17.01(0.06)$ & 359.32 & $17.05(0.06)$ & 365.73 & $17.26(0.06)$ & 374.40 & $17.14(0.07)$ \\
\hline 452.20 & $18.33(0.17)$ & 346.19 & $18.10(0.06)$ & 359.32 & $16.89(0.06)$ & 362.84 & $16.96(0.07)$ & 374.41 & $17.51(0.06)$ & 377.19 & $17.23(0.07)$ \\
\hline 456.92 & $18.24(0.17)$ & 366.43 & $18.15(0.11)$ & 362.84 & $17.22(0.08)$ & 365.10 & $17.13(0.07)$ & 377.20 & $17.42(0.05)$ & 380.91 & $17.16(0.07)$ \\
\hline 472.23 & $18.35(0.39)$ & 371.41 & $18.36(0.10)$ & 365.10 & $17.16(0.07)$ & 368.95 & $16.99(0.07)$ & 380.92 & $17.53(0.06)$ & 384.52 & $17.31(0.09)$ \\
\hline 477.55 & $18.22(0.26)$ & 393.66 & $18.35(0.11)$ & 368.95 & $17.04(0.07)$ & 374.40 & $17.17(0.07)$ & 384.51 & $17.35(0.06)$ & 226.52 & $16.89(0.05)$ \\
\hline 533.61 & $18.74(0.51)$ & 427.75 & $18.64(0.13)$ & 374.41 & $17.23(0.07)$ & 377.19 & $17.29(0.08)$ & 226.52 & $17.16(0.05)$ & 241.13 & $17.61(0.05)$ \\
\hline 552.75 & $18.26(0.25)$ & 447.41 & $19.10(0.22)$ & 377.20 & $17.20(0.08)$ & 380.92 & $17.25(0.07)$ & 241.12 & $17.77(0.06)$ & 248.48 & $17.83(0.06)$ \\
\hline 554.88 & $18.02(0.17)$ & 396.75 & $18.56(0.13)$ & 380.92 & $17.22(0.08)$ & 384.51 & $17.18(0.08)$ & 248.47 & $18.12(0.06)$ & 262.73 & $18.16(0.07)$ \\
\hline 557.37 & $18.52(0.17)$ & 399.25 & $18.62(0.14)$ & 384.51 & $17.22(0.09)$ & 226.52 & $16.62(0.05)$ & 262.73 & $18.38(0.07)$ & 272.68 & $17.88(0.07)$ \\
\hline 560.22 & $18.57(0.19)$ & 433.43 & $18.86(0.10)$ & 226.52 & $16.30(0.04)$ & 241.12 & $17.25(0.05)$ & 272.67 & $18.22(0.06)$ & 277.33 & $17.62(0.07)$ \\
\hline 563.21 & $18.82(0.40)$ & 452.19 & $18.91(0.14)$ & 241.12 & $16.63(0.05)$ & 248.47 & $17.43(0.06)$ & 277.36 & $17.87(0.05)$ & 284.57 & $17.32(0.05)$ \\
\hline \multirow[t]{23}{*}{569.44} & $18.42(0.27)$ & 456.91 & $18.91(0.13)$ & 248.47 & $16.78(0.05)$ & 262.73 & $17.55(0.06)$ & 298.64 & $17.54(0.05)$ & 298.65 & $17.11(0.05)$ \\
\hline & & 472.22 & $19.14(0.30)$ & 262.73 & $17.08(0.06)$ & 272.67 & $17.39(0.05)$ & 255.70 & $18.29(0.07)$ & 255.66 & $18.02(0.09)$ \\
\hline & & 477.55 & $19.03(0.21)$ & 272.88 & $17.01(0.07)$ & 277.35 & $17.15(0.05)$ & 305.49 & $17.34(0.05)$ & 305.50 & $17.03(0.06)$ \\
\hline & & 533.61 & $18.79(0.23)$ & 277.36 & $16.95(0.05)$ & 284.37 & $17.11(0.05)$ & 317.77 & $17.39(0.06)$ & 317.77 & $16.83(0.09)$ \\
\hline & & 552.75 & $19.01(0.19)$ & 284.37 & $16.97(0.05)$ & 298.64 & $17.01(0.05)$ & 330.19 & $17.28(0.05)$ & 330.19 & $16.97(0.05)$ \\
\hline & & 554.88 & $19.13(0.16)$ & 298.64 & $16.88(0.05)$ & 255.69 & $17.51(0.06)$ & 346.20 & $17.35(0.05)$ & 346.20 & $16.98(0.06)$ \\
\hline & & 557.36 & $19.09(0.12)$ & 255.69 & $16.91(0.05)$ & 284.77 & $17.12(0.06)$ & 371.42 & $17.41(0.05)$ & 371.43 & $17.25(0.07)$ \\
\hline & & 560.22 & $19.12(0.13)$ & 272.60 & $16.96(0.05)$ & 305.48 & $16.87(0.05)$ & 316.24 & $17.27(0.05)$ & 316.23 & $16.98(0.07)$ \\
\hline & & 563.21 & $19.04(0.21)$ & 284.77 & $16.95(0.05)$ & 317.73 & $17.02(0.06)$ & 319.77 & $17.43(0.06)$ & 319.76 & $17.13(0.07)$ \\
\hline & & 569.43 & $19.41(0.24)$ & 305.49 & $16.87(0.05)$ & 330.18 & $16.92(0.05)$ & 325.34 & $17.52(0.06)$ & 322.75 & $17.19(0.07)$ \\
\hline & & & & 317.77 & $16.94(0.06)$ & 346.19 & $17.02(0.05)$ & 328.53 & $17.30(0.05)$ & 325.33 & $17.22(0.07)$ \\
\hline & & & & 330.18 & $17.00(0.06)$ & 366.39 & $17.08(0.07)$ & 331.06 & $17.24(0.05)$ & 328.52 & $16.96(0.06)$ \\
\hline & & & & 346.19 & $16.97(0.05)$ & 371.41 & $17.16(0.06)$ & 334.58 & $17.32(0.06)$ & 331.05 & $17.03(0.07)$ \\
\hline & & & & 366.43 & $17.18(0.08)$ & 316.23 & $16.95(0.06)$ & 343.44 & $17.34(0.06)$ & 334.58 & $17.02(0.07)$ \\
\hline & & & & 371.41 & $17.17(0.07)$ & 321.23 & $16.98(0.06)$ & 349.22 & $17.27(0.06)$ & 343.43 & $17.06(0.06)$ \\
\hline & & & & 316.24 & $16.87(0.06)$ & 325.34 & $16.98(0.06)$ & 393.66 & $17.55(0.05)$ & 349.21 & $16.94(0.07)$ \\
\hline & & & & 319.76 & $17.01(0.06)$ & 328.53 & $16.78(0.06)$ & 427.75 & $18.16(0.07)$ & 393.67 & $17.37(0.07)$ \\
\hline & & & & 325.34 & $16.92(0.06)$ & 331.05 & $16.82(0.06)$ & 447.41 & $18.06(0.07)$ & 427.75 & $17.83(0.08)$ \\
\hline & & & & 328.53 & $16.86(0.05)$ & 334.58 & $16.80(0.06)$ & 396.76 & $17.55(0.06)$ & 447.41 & $17.87(0.08)$ \\
\hline & & & & 331.05 & $16.86(0.06)$ & 343.43 & $16.93(0.06)$ & 399.25 & $17.41(0.06)$ & 396.76 & $17.31(0.07)$ \\
\hline & & & & 334.58 & $16.97(0.06)$ & 349.21 & $16.88(0.06)$ & 429.65 & $18.06(0.09)$ & 399.25 & $17.29(0.07)$ \\
\hline & & & & 343.43 & $17.03(0.06)$ & 393.66 & $17.27(0.06)$ & 433.51 & $18.15(0.09)$ & 429.65 & $17.83(0.10)$ \\
\hline & & & & 349.21 & $16.98(0.06)$ & 427.74 & $17.59(0.07)$ & 437.32 & $18.16(0.08)$ & 435.30 & $17.84(0.08)$ \\
\hline
\end{tabular}


Table 2

(Continued)

\begin{tabular}{|c|c|c|c|c|c|c|c|c|c|c|c|}
\hline Date & $v(\mathrm{mag})$ & Date & $b$ (mag) & Date & $u$ (mag) & Date & $w 1$ (mag) & Date & $w 2(\mathrm{mag})$ & Date & $m 2$ (mag) \\
\hline & & & & 427.75 & $17.73(0.09)$ & 396.75 & $17.20(0.07)$ & 454.22 & $18.17(0.08)$ & 454.22 & $17.90(0.09)$ \\
\hline & & & & 447.41 & $18.00(0.12)$ & 399.25 & $17.27(0.07)$ & 457.64 & $18.19(0.10)$ & 456.09 & $17.89(0.09)$ \\
\hline & & & & 399.25 & $17.33(0.07)$ & 433.50 & $17.76(0.10)$ & 465.56 & $18.17(0.07)$ & 470.33 & $17.92(0.07)$ \\
\hline & & & & 431.64 & $17.85(0.10)$ & 437.32 & $17.71(0.09)$ & 470.34 & $18.19(0.06)$ & 472.23 & $18.05(0.16)$ \\
\hline & & & & 437.32 & $17.75(0.11)$ & 450.03 & $17.82(0.12)$ & 472.23 & $18.18(0.13)$ & 475.67 & $17.94(0.08)$ \\
\hline & & & & 456.91 & $17.81(0.08)$ & 456.07 & $17.83(0.08)$ & 480.30 & $18.39(0.07)$ & 487.48 & $18.29(0.15)$ \\
\hline
\end{tabular}

Note.

${ }^{\mathrm{a}}$ Dates are in MJD - 57000 (days).

(This table is available in machine-readable form.)

\section{References}

Alexander, K. D., Berger, E., Guillochon, J., Zauderer, B. A., \& Williams, P. K. G. 2016, ApJL, 819, L25

Allington-Smith, J., et al. 1994, PASP, 106, 983

Berger, E., Zauderer, A., Pooley, G. G., et al. 2012, ApJ, 748, 36

Bersten, M. C., Benvenuto, O. G., Orellana, M., \& Nomoto, K. 2016, ApJL, 817, L8

Bonnerot, C., Rossi, E. M., Lodato, G., \& Price, D. J. 2016, MNRAS, 455, 2253

Branch, D., Jeffery, D. J., Blaylock, M., \& Hatano, K. 2000, PASP, 112, 217

Breeveld, A. A., Landsman, W., Holland, S. T., et al. 2011, AIP Conf. Ser 1358, ed. J. E. McEnery, J. L. Racusin, \& N. Gehrels (San Francisco, CA: ASP), 373

Brown, J. S., Holoien, W.-S., Auchettl, T., et al. 2016a, arXiv

Brown, P. J., Holland, S. T., Immler, S., et al. 2009, AJ, 137, 4517

Brown, P. J., Yang, Yi., Cooke, J., et al. 2016b, ApJ, 828, 3

Bruzual, G., \& Charlot, S. 2003, MNRAS, 344, 1000

Burrows, D. N., Hill, J. E., Nousek, J. A., et al. 2005, SSRv, 120, 165

Cenko, S. B., Krimm, H. A., Horesh, A., et al. 2012, ApJ, 753, 77

Chabrier, G. 2003, PASP, 115, 763

Chandra, P., \& Frail, D. A. 2012, ApJ, 746, 156

Chatzopoulos, E., \& Wheeler, J. C. 2012, ApJ, 760, 154

Chatzopoulos, E., Wheeler, J. C., Vinko, J., et al. 2016, ApJ, 828, 94

Chatzopoulos, E., Wheeler, J. C., Vinko, J., Horvath, Z. L., \& Nagy, A. 2013, ApJ, 773, 76

Chevalier, R. A., \& Fransson, C. 2006, ApJ, 651, 381

Chomiuk, L., Chornock, R., Soderberg, A. M., et al. 2011, ApJ, 743, 114

Chomiuk, L., Soderberg, A., Margutti, R., et al. 2012, ATel, 3931

Chornock, R., Berger, E., Gezari, S., et al. 2014, ApJ, 780, 44

Crenshaw, D. M., Kraemer, S. B., Hutchings, J. B., et al. 2000, ApJL, 545, L27

Dai, L., McKinney, J. C., \& Miller, M. C. 2015, ApJL, 812, L39

Dai, Z. G., Wang, S. Q., Wang, J. S., Wang, L. J., \& Yu, Y. W. 2016, ApJ, 817,132

Dong, S., Shappee, B. J., Prieto, J. L., et al. 2016, Sci, 351, 257

Drout, M. R., Chornock, R., Soderberg, A. M., et al. 2014, ApJ, 794, 23

Dwarkadas, V. V., \& Gruszko, J. 2012, MNRAS, 419, 1515

Filippenko, A. V. 1982, PASP, 94, 715

Gabel, J. R., Crenshaw, D. M., Kraemer, S. B., et al. 2003, ApJ, 595, 120

Gal-Yam, A. 2012, Sci, 337, 927

Gezari, S., Chornock, R., Rest, A., et al. 2012, Natur, 485, 217

Godoy-Rivera, D., Stanek, K. Z., Kochanek, C. S., et al. 2016, MNRAS, 466, 1428

Guillochon, J., \& Ramirez-Ruiz, E. 2013, ApJ, 767, 25

Guillochon, J., \& Ramirez-Ruiz, E. 2015, ApJ, 809, 166

Hayasaki, K., Stone, N., \& Loeb, A. 2016, MNRAS, 461, 3760

Holoien, T. W.-S., Kochanek, C. S., Prieto, J. L., et al. 2016a, MNRAS, 463, 3813

Holoien, T. W.-S., Kochanek, C. S., Prieto, J. L., et al. 2016b, MNRAS, 455, 2918

Holoien, T. W.-S., Prieto, J. L., Bersier, D., et al. 2014, MNRAS, 445, 3263

Jeffery, D. J., Ketchum, W., Branch, D., et al. 2007, ApJS, 171, 493

Kalberla, P. M. W., Burton, W. B., Hartmann, D., et al. 2005, A\&A, 440, 775

Kasen, D., \& Bildsten, L. 2010, ApJ, 717, 245

Kennicutt, R. C., Jr. 1998, ARA\&A, 36, 189
Kesden, M. 2012, PhRvD, 86, 064026

Kewley, L. J., Groves, B., Kauffmann, G., \& Heckman, T. 2006, MNRAS, 372,961

Kim, D.-W., \& Fabbiano, G. 2004, ApJ, 611, 846

Kochanek, C. S. 2016, MNRAS, 458, 127

Komossa, S. 2015, JHEAp, 7, 148

Kool, E. C., Ryder, S. D., Stockdale, C. J., et al. 2015, ATel, 8388

Kozyreva, A., Hirschi, R., Blinnikov, S., \& den Hartogh, J. 2016, MNRAS, 459, L21

Kraemer, S. B., Crenshaw, D. M., George, I. M., et al. 2002, ApJ, 577, 98

Kriek, M., van Dokkum, P. G., Labbé, I., et al. 2009, ApJ, 700, 221

Leaman, J., Li, W., Chornock, R., \& Filippenko, A. V. 2011, MNRAS, 412, 1419

Leloudas, G., Fraser, M., Stone, N. C., et al. 2016, NatAS, 1, 2

Levan, A. J., Read, A. M., Metzger, B. D., Wheatley, P. J., \& Tanvir, N. R. 2013, ApJ, 771, 136

Loeb, A., \& Ulmer, A. 1997, ApJ, 489, 573

Lunnan, R., Chornock, R., Berger, E., et al. 2014, ApJ, 787, 138

Lunnan, R., Chornock, R., Berger, E., et al. 2015, ApJ, 804, 90

Mangano, V., Burrows, D. N., Sbarufatti, B., \& Cannizzo, J. K. 2016, ApJ, 817, 103

Maran, S. P., Crenshaw, D. M., Mushotzky, R. F., et al. 1996, ApJ, 465, 733 Margutti, R., Milisavljevic, D., Soderberg, A. M., et al. 2014a, ApJ, 780, 21

Margutti, R., Milisavljevic, D., Soderberg, A. M., et al. 2014b, ApJ, 797, 107

Margutti, R., Soderberg, A. M., Wieringa, M. H., et al. 2013a, ApJ, 778, 18

Margutti, R., Zaninoni, E., Bernardini, M. G., et al. 2013b, MNRAS, 428, 729

Melchior, P., Drlica-Wagner, A., Bechtol, K., Rykoff, E., Hartley, W. \& Dark Energy Survey Collaboration 2015, ATel, 7843

Metzger, B. D., Margalit, B., Kasen, D., \& Quataert, E. 2015, MNRAS, 454, 3311

Metzger, B. D., \& Stone, N. C. 2016, MNRAS, 461, 948

Metzger, B. D., Vurm, I., Hascoët, R., \& Beloborodov, A. M. 2014, MNRAS, 437, 703

Miller, J. M., Kaastra, J, S., Miller, M. C., et al. 2015, Natur, 526, 542

Nicholl, M., Berger, E., Smartt, S. J., et al. 2016, ApJ, 826, 39

Pasham, D. R., Cenko, S. B., Levan, A. J., et al. 2015, ApJ, 805, 68

Pastorello, A., Smartt, S. J., Botticella, M. T., et al. 2010, ApJL, 724, L16

Perley, D. A., Quimby, R. M., Yan, L., et al. 2016, ApJ, 830, 13

Quimby, R. M., Kulkarni, S. R., Kasliwal, M. M., et al. 2011, Natur, 474, 487 Rees, M. J. 1988, Natur, 333, 523

Roth, N., Kasen, D., Guillochon, J., \& Ramirez-Ruiz, E. 2016, ApJ, 827, 3

Schlafly, E. F., \& Finkbeiner, D. P. 2011, ApJ, 737, 103

Spitkovsky, A. 2006, ApJL, 648, L51

Stone, N., Sari, R., \& Loeb, A. 2013, MNRAS, 435, 1809

Sukhbold, T., \& Woosley, S. E. 2016, ApJL, 820, L38

Thomas, R. C., Nugent, P. E., \& Meza, J. C. 2011, PASP, 123, 237

van Eerten, H., Zhang, W., \& MacFadyen, A. 2010, ApJ, 722, 235

van Putten, M. H. P. M., \& Della Valle, M. 2017, MNRAS, 464, 3219

van Velzen, S., Frail, D. A., Körding, E., \& Falcke, H. 2013, A\&A, 552, A5

van Velzen, S., Anderson, G. E., Stone, N. C., et al. 2016, Sci, 351, 62

Woosley, S. E. 2010, ApJL, 719, L204

Yan, L., Quimby, R., Ofek, E., et al. 2015, ApJ, 814, 108

Yaron, O., \& Gal-Yam, A. 2012, PASP, 124, 668

Zauderer, B. A., Berger, E., Margutti, R., et al. 2013, ApJ, 767, 152 